\title{
Accelerated Degradation Tests Planning With Competing Failure Modes
}

\author{
Xiujie Zhao ${ }^{\mathrm{a}^{* \dagger}}$, Jianyu $\mathrm{Xu}^{\mathrm{b}, \mathrm{c}}$ and Bin $\mathrm{Liu}^{\mathrm{a}}$ \\ ${ }^{a}$ Department of Systems Engineering and Engineering Management \\ City University of Hong Kong, Hong Kong \\ ${ }^{b}$ City University of Hong Kong Shenzhen Research Institute, Shenzhen, China \\ 'University of Chinese Academy of Sciences, Beijing, China
}

\begin{abstract}
Accelerated degradation tests (ADT) have been widely used to assess the reliability of products with long lifetime. For many products, environmental stress not only accelerates their degradation rate but also elevates the probability of traumatic shocks. When random traumatic shocks occur during an ADT, it is possible that the degradation measurements cannot be taken afterward, which brings challenges to reliability assessment. In this paper, we propose an ADT optimization approach for products suffering from both degradation failures and random shock failures. The degradation path is modeled by a Wiener process. Under various stress levels, the arrival process of random shocks is assumed to follow a non-homogeneous Poisson process. Parameters of acceleration models for both failure modes need to be estimated from the ADT. Three common optimality criteria based on the Fisher information are considered and compared to optimize the ADT plan under a given number of test units and a pre-determined test duration. Optimal two- and three-level optimal ADT plans are obtained by numerical methods. We use the general equivalence theorems to verify the global optimality of ADT plans. A numerical example is presented to illustrate the proposed methods. The result shows that the optimal ADT plans in the presence of random shocks differ significantly from the traditional ADT plans. Sensitivity analysis is carried out to study the robustness of optimal ADT plans with respect to the changes in planning input.
\end{abstract}

Keywords: accelerated degradation tests, competing failure modes, degradation modeling, optimal design, Fisher information, reliability assessment

\footnotetext{
${ }^{*}$ Corresponding author: xiujizhao2-c@my.cityu.edu.hk

$\dagger$ The work described in this paper was partially supported by a theme-based project grant (T32-101/15-R) of University Grants Council of Hong Kong, and a Key Project (71532008) supported by National Natural Science Foundation of China.
} 


\section{Acronyms and Abbreviations}

ADT

GET

MLE

PF0

PF1

RE

$T_{D}$

$D$

$B(\cdot)$

$\mathcal{J} \mathcal{G}(a, b)$

$\theta$

$\hat{\boldsymbol{\theta}}$

$t_{p}$

$\Delta t$

$\eta(x)$

$J$

$N$

$x_{j}, j=1, \ldots, J$

$\pi_{j}, j=1, \ldots, J$

$N_{j}, j=1, \ldots, J$

$\mathbf{I}(\boldsymbol{\theta})$
Accelerated degradation test

General equivalence theorems

Maximum likelihood estimation

Proportion of failure under use condition

Proportion of failure under the maximum stress

Relative efficiency

\section{Notation}

Degradation path of the test product

Lifetime of the test product

Degradation threshold

Standard Brownian motion

Inverse Gaussian distribution with mean $a$ and scale $b$

True parameters

MLE of $\boldsymbol{\theta}$

$100 p$-th lifetime percentile

Inspection interval in the ADT

Drift parameter under standardized stress $x$

Number of stress levels

Number of test units

Level of the $j$-th stress

Proportion of test units allocated to the $j$-th stress

Number of test units allocated to the $j$-th stress

Fisher information matrix 


$\begin{array}{ll}\Phi(\cdot), \phi(\cdot) & \text { CDF and PDF of the standard normal distribution } \\ \operatorname{tr}(\mathbf{X}) & \text { Trace of matrix } \mathbf{X} \\ \operatorname{det}(\mathbf{X}) & \text { Determinant of } \mathbf{X} \\ \mathrm{E}(X) & \text { Expectation of random variable } X \\ \operatorname{AVar}(X) & \text { Asymptotic variance of } X \\ \mathcal{P}, \mathcal{P}^{*} & \text { Two-level test plan and optimal plan } \\ \mathcal{C} \mathcal{P}_{i}^{*} & \text { The optimal compromise plan }\end{array}$

\section{Introduction}

Accelerated reliability tests are commonly used to assess the reliability of new products, especially those with extremely long lifespan under field use. In such tests, the products are exposed to elevated stress conditions, such as higher temperature, pressure, humidity, or a combination of them. Data analysis and optimal planning of accelerated tests have drawn considerable attention from reliability researchers and engineers, who desire to predict the reliability as precise as possible through a more economical approach. Test information from a well-planned accelerated reliability test can provide useful guidance for maintenance scheduling and warranty prediction [1], [2]. For an overview, see Elsayed [3].

Inferences of lifetime distribution from accelerated life tests (ALT) is becoming very challenging because many highly reliable products have none or very few failures even under elevated stresses in a reasonable test duration. In such situations, we can resort to accelerated degradation tests (ADT) if the product has one or more measurable quality characteristics (QC) that can be modeled as degradation processes [4]. Instead of observing the failure times as in ALT, degradation levels of test units are measured periodically in ADT. The planning of ADT or other types of degradation test, such as step-stress ADT (SSADT) [5] and accelerated destructive degradation tests (ADDT) [6] have proved to be efficient in enhancing the accuracy of reliability assessment and saving experimental resources. The optimal ADT plans based on Wiener processes [7], [8], gamma processes [9]-[11], and inverse Gaussian processes [12] have been intensely studied in the literature. 
Most existing works on optimal ALT/ADT planning assumed that there was only one failure mode. However, many products have more than one failure modes. Neglect of any failure mode may significantly influence the optimality of reliability test plans and therefore the prediction accuracy of lifetime, thus it is necessary to consider multiple failure modes when planning accelerated tests. Bai and Chun [13] discussed the optimal simple step-stress ALT (SSALT) plans with independent competing causes. Afterward, Pascual [14], [15] studied the ALT planning by considering independent Weibull or lognormal competing risks. For repairable systems, Liu and Tang [16] used a Bayesian D-optimality criterion to optimize ALT plans with independent risks, and an extension to SSALT can be found in Liu and Qiu [17]. Similar ideas have also been discussed for ALT with multiple stresses [18] and dependent failure modes [19].

Although there are numerous studies on ALT planning with more than one failure modes, fewer studies have addressed the ADT modeling and planning with multiple failure modes. Ye et al. [20] developed a burn-in planning method by differentiating normal and mortality failure modes. The optimal two-variable ADT planning method for gamma processes was discussed in Tsai et al. [10]. Li and Jiang [21] proposed a SSADT planning method with independent stochastic degradation processes. Furthermore, SSADT planning problem with two dependent gamma processes was studied by Pan and Sun [22]. Haghighi and Bae modeled and analyzed linear degradation data and traumatic failures with competing risks in an SSADT experiment with the cumulative exposure model [23]. Nevertheless, to our knowledge, none of the studies in literature has considered both degradation and shock failures in the ADT planning problem, although the joint modeling of degradation and random shocks as well as related maintenance/warranty problems have been very popular in recent literature [24]-[26]. It is common that either performance degradation or random traumatic shocks could lead to failures. Generally, performance degradation is due to the natural aging and usage of a product, and if the performance degrades to an unsatisfactory level, the product is deemed failed although it may still work. For example, an LED lamp are commonly regarded failed if its light intensity drops to a certain level. On the other hand, traumatic shocks are more likely to be caused by external events that influence the whole system, such as the sudden change in currency and voltage for electronic devices. The shocks lead to immediate product failures. During an ADT, the degradation measurability can be influenced by random shocks. Regarding the LED lamp example, if a lamp suddenly goes out during an ADT, its brightness cannot be tracked after the failure. In this situation, test planners need to consider the possibility that 
increasing number of random shocks under elevated stress conditions significantly decreases the test information due to the partial missing of degradation measurements. Figure 1 shows an illustration of such cases, where each 10 test units are allocated to low- and high-level stresses, respectively. The measurements from high-level stress provide more information on the acceleration relationship of degradation, but the stress also leads to more shock failures during the test. As is shown there are only three test units that survive to the end of the test and provide full degradation information during the test. In contrast, there is no shock failure for the units under low-level stress, and the degradation measurements are complete, yet the degradation increase is not significant so that the inference of the degradation rate can be greatly influenced by random noises. In previous ADT studies, test planners usually took full advantage of the highest possible stress to obtain measurements with high degradation rate as long as it is believed that the degradation mechanism remains the same for the highest stress [27]. However, if random shocks are taken into account, higher stress may lead to much more shock failures in the test process and the number of degradation measurements become considerably less, which will cause loss in data to estimate unknown parameters and predict lifetime under use condition.

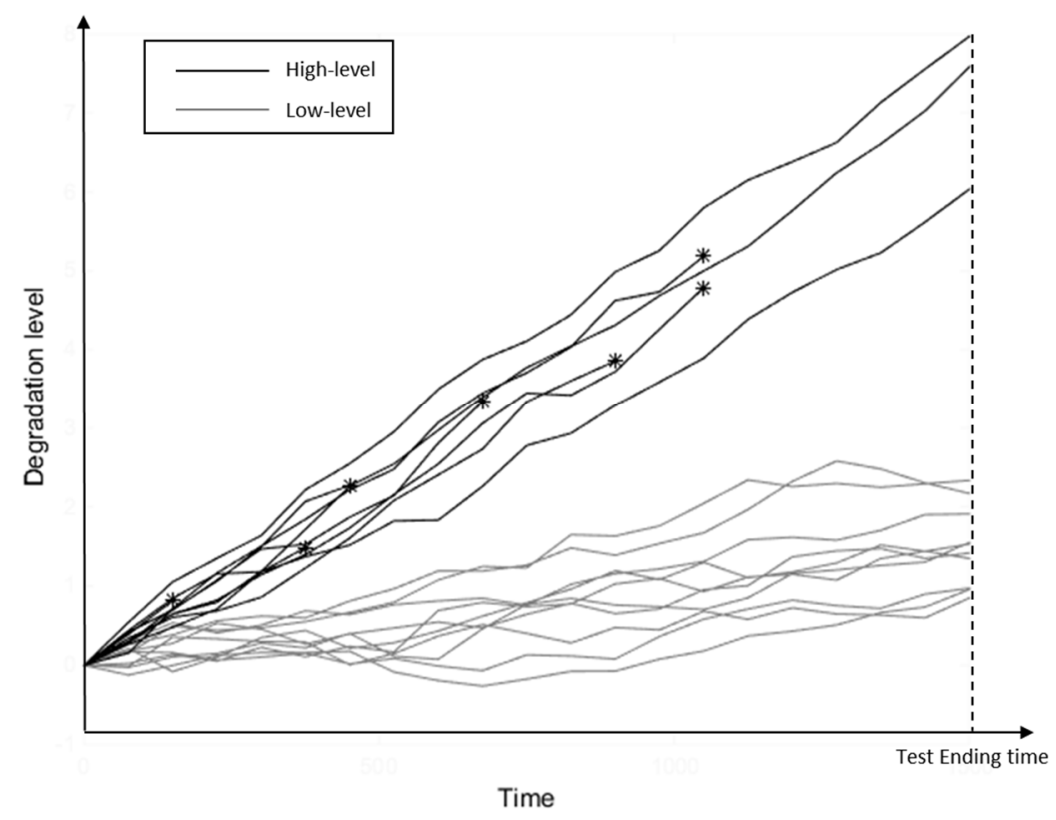

Figure 1 Stress-related shocks in ADT experiment

Considering the shock issue in ADTs, we propose an optimal ADT planning approach with competing failure modes in this paper. The product to be tested is assumed to suffer from both degradation and shock failures. Both failure modes are accelerated by a common experimental 
factor during the test. If a test unit fails due to a shock, the degradation cannot be tracked afterward. The random shocks are assumed to be independent of the degradation levels and arrive by a Poisson process with intensity determined by the stress. The objective is to obtain the optimal ADT plans under three common optimality criteria. Our results show the necessity of incorporating random shocks into the model by investigating the optimal ADT plans under various failure assumptions.

The rest of the paper is organized as follows. Section 2 presents the ADT modeling with competing failure modes and derives the expression of the lifetime distribution. Planning criteria and optimization problem formulation are discussed in Section 3. In Section 4, a numerical example is provided to illustrate the application of the proposed planning method, followed by a sensitivity analysis in Section 5. Finally, Section 6 concludes the paper and discusses areas for future research.

\section{ADT modeling with competing failure modes}

\subsection{Joint modeling of degradation and random shocks}

Stochastic models have been widely used to model degradations because of their clear physical explanations and tractable mathematical properties [28]. We use a Wiener process to model the degradation in this paper. The model proposed in this paper can also be applied to other degradation processes, such as gamma processes and inverse Gaussian processes. It is assumed that the product suffers from a measurable Wiener degradation, denoted by $Y(t)$. A degradation failure is deemed to occur when $Y(t)$ hits a pre-determined threshold $D$, thus the failure time is the first passage time (FPT) of $Y(t)$ to threshold $D$, denoted by $T_{D}$. The basic Wiener process is a drifted Brownian motion as follows:

$$
Y(t)=\eta \Lambda(t)+\sigma B(\Lambda(t))
$$

where $\eta>0$ is the drift parameter, $\sigma$ is the diffusion parameter, $\Lambda(t)$ is a time scale function to describe the nonlinearity degradation path, and $B(\cdot)$ is the standard Brownian motion. As in many previous literature of reliability modeling and testing [7], $\Lambda(t)=t$ is assumed, i.e., the degradation path is linear with respect to time. Under this assumption, $T_{D}$ follows an inverse Gaussian (IG) distribution with mean $D / \eta$ and scale $D^{2} / \sigma^{2}$, that is, $T_{D} \sim \mathcal{J} \mathcal{G}\left(D / \eta, D^{2} / \sigma^{2}\right)$, of which the PDF is given by 


$$
f_{T_{D}}(t ; D, \eta, \sigma)=\left[\frac{D^{2}}{2 \pi \sigma^{2} t^{3}}\right]^{1 / 2} \exp \left\{\frac{-(\eta t-D)^{2}}{2 \sigma^{2} t}\right\}
$$

and the CDF is given by

$$
F_{T_{D}}(t ; D, \eta, \sigma)=\Phi\left[\sqrt{\frac{1}{\sigma^{2} t}}(\eta t-D)\right]+\exp \left\{\frac{2 \eta D}{\sigma^{2}}\right\} \Phi\left[-\sqrt{\frac{1}{\sigma^{2} t}}(\eta t+D)\right]
$$

where $\Phi$ is the CDF of standard normal distribution. In addition to degradation risk, random traumatic shocks may also occur to the product of interest. As this type of failure is sudden and immediate, we call it a "shock failure". We assume that shock failures are independent of degradation failures. It is noted that the assumption of independency is valid if the two failure modes do not have interactive effect. Take LED lamps as an example, the natural internal performance degradation in light intensity and sudden shocks caused by the voltage or currency have no direct correlation because the shocks are mainly due to external reasons, however, they may be both influenced by environmental stresses such as temperature and humidity. The time between random shocks can be modeled by an exponential distribution with mean $1 / \lambda$. Denote the time to a shock failure as $T_{S}$, of which PDF and CDF are given by

$$
f_{T_{S}}(t ; \lambda)=\lambda \exp (-\lambda t), \quad F_{T_{S}}(t ; \lambda)=1-\exp (-\lambda t)
$$

The lifetime of the product, denoted by $T$, is determined by either degradation failure or shock failure times, whichever comes first, i.e., $T=\min \left\{T_{D}, T_{S}\right\}$. We can derive the CDF of $T$ as follows:

$$
\begin{aligned}
F_{T}(t ; D, \eta, \sigma, \lambda) & =\operatorname{Pr}\left(\min \left\{T_{D}, T_{S}\right\} \leq t\right) \\
= & 1-\left[1-F_{T_{D}}(t ; D, \eta, \sigma)\right]\left[1-F_{T_{S}}(t ; \lambda)\right] \\
= & 1 \\
& -\exp (-\lambda t)\left\{1-\Phi\left[\sqrt{\frac{1}{\sigma^{2} t}}(\eta t-D)\right]\right. \\
& \left.-\exp \left(\frac{2 \eta D}{\sigma^{2}}\right) \Phi\left[-\sqrt{\frac{1}{\sigma^{2} t}}(\eta t+D)\right]\right\}
\end{aligned}
$$

and the PDF is given by 


$$
\begin{aligned}
f_{T}(t ; D, \eta, \sigma, \lambda) & =\frac{\partial F_{T}(t ; D, \eta, \sigma, \lambda)}{\partial t} \\
& =\lambda \exp (-\lambda t)\left\{1-\Phi\left[\sqrt{\frac{1}{\sigma^{2} t}}(\eta t-D)\right]\right. \\
& \left.-\exp \left(\frac{2 \eta D}{\sigma^{2}}\right) \Phi\left[-\sqrt{\frac{1}{\sigma^{2} t}}(\eta t+D)\right]\right\} \\
& -\exp (-\lambda t)\left\{-\frac{1}{2 \sigma}\left(\frac{\eta}{\sqrt{t}}+\frac{D}{\sqrt{t^{3}}}\right) \phi\left[\sqrt{\frac{1}{\sigma^{2} t}}(\eta t-D)\right]\right. \\
& \left.+\frac{1}{2 \sigma} \exp \left(\frac{2 \eta D}{\sigma^{2}}\right)\left(\frac{\eta}{\sqrt{t}}-\frac{D}{\sqrt{t^{3}}}\right) \phi\left[-\sqrt{\frac{1}{\sigma^{2} t}}(\eta t+D)\right]\right\}
\end{aligned}
$$

where $\phi(\cdot)$ is the PDF of standard normal distribution.

\subsection{ADT data modeling}

Suppose a total number of $N$ test units are provided for the ADT and assume that there is only one stress factor in the test. Let $s_{0}$ and $s_{H}$ be the stress under use condition and maximum stress level allowed in the test, respectively. There are totally $J$ stress levels in the test, denoted by $s_{1}, \ldots, s_{J}$. Let $N_{j}$ be the number of test units allocated to the $j$-th stress level, $j=1, \ldots, J$,

and $\sum_{j=1}^{J} N_{j}=N$. We assume that the stress simultaneously influences the degradation rate and the intensity of random shocks. Firstly, we standardize $s_{j}$ as follows:

$$
x_{l}=\left\{\begin{array}{lr}
\frac{\ln s_{j}-\ln s_{0}}{\ln s_{H}-\ln s_{0}} & \text { for the power law relation } \\
\frac{1 / s_{0}-1 / s_{l}}{1 / s_{0}-1 / s_{H}} & \text { for the Arrhenius relation } \\
\frac{s_{j}-s_{0}}{s_{H}-s_{0}} & \text { for the exponential relation }
\end{array}\right.
$$

For the details of the acceleration model and standardization, see Jakob et al. [29] and Lim and Yum [7, Sec. 3.1]. The standardization yields $x_{0}=0$ and $x_{H}=1$. Denote $\eta(x)$ as the drift parameter of the Wiener degradation process under stress $x$, and it is given by

$$
\eta(x)=\exp \left(\alpha_{1}+\beta_{1} x\right)
$$

The shock failure rate $\lambda$ is also influenced by the elevated stress. Let $\lambda(x)$ be the shock failure rate under stress $x$. We have 


$$
\lambda(x)=\exp \left(\alpha_{2}+\beta_{2} x\right)
$$

The unknown parameters in the model are $\boldsymbol{\theta}=\left(\alpha_{1}, \beta_{1}, \sigma, \alpha_{2}, \beta_{2}\right)^{\prime}$. We assume that the total number of test units $N$, the test duration $t_{C}$, and the measurement time interval $\Delta t$ are predetermined. Therefore, the decision variables in the problem are the stress levels and the proportion of test units allocated to each level. Due to the presence of shock failures, some degradation measurements for a certain test unit cannot be obtained, i.e., the degradation measures are "censored" by a shock failure. Assume $t_{C}=K \Delta t$, i.e., there are $K$ measurements for a test unit if it survives until the end of the test. The shock failure is not self-announcing and can only be revealed by inspection. As we only have interval inspections, the shock failures are interval censored. The probability that the $i$-th test under stress $s_{j}$ has a shock failure between measurement $k-1$ and $k(1 \leq k \leq K)$ is given by

$$
\begin{aligned}
p_{i j k}^{(S)}=\operatorname{Pr}\left[(k-1) \Delta t<T_{S} \leq k \Delta t\right] & \\
& =\exp \left[-\lambda\left(x_{j}\right)(k-1) \Delta t\right]-\exp \left[-\lambda\left(x_{j}\right) k \Delta t\right]
\end{aligned}
$$

and the probability that the $i$-th test unit under stress $s_{j}$ survives from shock failures during the test is

$$
r_{i j}^{(S)}=\operatorname{Pr}\left[T_{S}>K \Delta t\right]=\exp \left[-\lambda\left(x_{j}\right) K \Delta t\right]
$$

Denote $Y_{i j}(\cdot)$ as the degradation process for the $i$-th test units under stress $s_{j}$. By the properties of Wiener process, the increment between the $k-1$ and $k$-th inspection interval, $Y_{i j k}$, follows a normal distribution. For $k=1, \ldots, K$ :

$$
Y_{i j k}=Y_{i j}(k \Delta t)-Y_{i j}((k-1) \Delta t) \sim \mathcal{N}\left(\eta\left(x_{j}\right) \Delta t, \sigma^{2} \Delta t\right)
$$

A realization of $Y_{i j k}$ is denoted as $y_{i j k}$. Let $\delta_{i j}$ be the integer variables describing the occurrence of shock failures, for the $i$-th test unit under $s_{j}$, and

$$
\delta_{i j}= \begin{cases}k \text { for } 1 \leq k \leq K & \text { if the test unit fails between } k-1 \text { and } k \text {-th inspection } \\ K+1 & \text { if the test unit survives through the test }\end{cases}
$$

The likelihood function of $\boldsymbol{\theta}$ is given by 


$$
=\prod_{j=1}^{J} \prod_{i=1}^{N_{j}}\left\{\left[\begin{array}{c}
{\left[p_{i j 1}^{(S)}\right]^{1_{\left\{\delta_{i j}=1\right\}}}\left[p_{i j \delta_{i j}}^{(S)} \prod_{k=1}^{\delta_{i j}-1} \frac{1}{\sigma \sqrt{\Delta t}} \phi\left(\frac{y_{i j k}-\eta\left(x_{j}\right) \Delta t}{\sqrt{\sigma^{2} \Delta t}}\right)\right]^{1_{\left\{2 \leq \delta_{i j} \leq K\right\}}}} \\
\times\left[r_{i j}^{(S)} \prod_{k=1}^{K} \frac{1}{\sigma \sqrt{\Delta t}} \phi\left(\frac{y_{i j k}-\eta\left(x_{j}\right) \Delta t}{\sqrt{\sigma^{2} \Delta t}}\right)\right]^{1_{\left\{\delta_{i j}=K+1\right\}}}
\end{array}\right\}\right.
$$

where $1_{\{\cdot\}}$ is the indicator function. The log-likelihood function can be expressed as

$$
\begin{aligned}
& \ell(\boldsymbol{\theta})=\sum_{j=1}^{J} \sum_{i=1}^{N_{j}}\left\{1_{\left\{\delta_{i j}=1\right\}} \log p_{i j 1}^{(S)}\right. \\
& +1_{\left\{2 \leq \delta_{i j} \leq K\right\}}\left[\log p_{i j \delta_{i j}}^{(S)}+\sum_{k=1}^{\delta_{i j}-1} \log \frac{1}{\sigma \sqrt{\Delta t}} \phi\left(\frac{y_{i j k}-\eta\left(x_{j}\right) \Delta t}{\sqrt{\sigma^{2} \Delta t}}\right)\right] \\
& \left.+1_{\left\{\delta_{i j}=K+1\right\}}\left[\log r_{i j}^{(S)}+\sum_{k=1}^{K} \log \frac{1}{\sigma \sqrt{\Delta t}} \phi\left(\frac{y_{i j k}-\eta\left(x_{j}\right) \Delta t}{\sqrt{\sigma^{2} \Delta t}}\right)\right]\right\} \\
& =\sum_{j=1}^{J} \sum_{i=1}^{N_{j}}\left\{1_{\left\{\delta_{i j}=1\right\}} \log \left\{1-\exp \left[-\lambda\left(x_{j}\right) \Delta t\right]\right\}\right. \\
& +1_{\left\{2 \leq \delta_{i j} \leq K\right\}}\left[\log \left\{\exp \left[-\lambda\left(x_{j}\right)\left(\delta_{i j}-1\right) \Delta t\right]-\exp \left[-\lambda\left(x_{j}\right) \delta_{i j} \Delta t\right]\right\}\right. \\
& \left.-\frac{\delta_{i j}-1}{2}\left(\log 2 \pi+\log \sigma^{2}+\log \Delta t\right)-\sum_{k=1}^{\delta_{i j}-1} \frac{\left[y_{i j k}-\eta\left(x_{j}\right) \Delta t\right]^{2}}{2 \sigma^{2} \Delta t}\right] \\
& +1_{\left\{\delta_{i j}=K+1\right\}}\left[-\lambda\left(x_{j}\right) K \Delta t-\frac{K}{2}\left(\log 2 \pi+\log \sigma^{2}+\log \Delta t\right)\right. \\
& \left.\left.-\sum_{k=1}^{K} \frac{\left[y_{i j k}-\eta\left(x_{j}\right) \Delta t\right]^{2}}{2 \sigma^{2} \Delta t}\right]\right\}
\end{aligned}
$$

The log-likelihood function can be maximized to obtain the MLE of unknown parameters,

denoted by $\hat{\boldsymbol{\theta}}$. By plugging $\hat{\boldsymbol{\theta}}$ into Eq. (5) or (6), we can obtain the maximum likelihood estimated lifetime distribution for the product. 


\section{Optimization of ADT plans}

\subsection{Planning criteria and the Fisher information}

To design a test with a relatively large sample size, test planners can adopt a particular asymptotic criterion based on the Fisher information of unknown parameters. Several common criteria include [30]:

- $\quad[\mathrm{C} 1]$ : $D$-optimality: to maximize the determinant of Fisher information matrix;

- [C2]: $A$-optimality: to minimize the trace of the first order approximation of variancecovariance matrix of the MLEs of unknown parameters, which is equivalent to minimizing the sum of the eigenvalues of the inverse Fisher information matrix;

- [C3]: $V$-optimality: to minimize the asymptotic variance of the estimated $100 p$-th percentile of the lifetime distribution, i.e., $\mathrm{A} \operatorname{Var}\left(\hat{t}_{p}\right)$.

For convenience, we use [C1], [C2] and [C3] to represent $D$-, $A$ - and $V$-optimality criterion, respectively. [C1] and [C2] concern more about the overall statistical variability of parameter estimates, whereas [C3] considers the accuracy of a specific lower lifetime percentile estimate that can be directly used to guide maintenance and warranty.

The optimization through any of above criteria needs the derivation of Fisher information matrix, which can provide a good approximation for the inverse of the large-sample asymptotic variance-covariance matrix of $\boldsymbol{\theta}$. The Fisher information is obtained by taking the expectation of negative second derivatives of $\ell(\boldsymbol{\theta})$. In our model, the Fisher information matrix $\mathbf{I}(\boldsymbol{\theta})$ can be expressed as

$\mathbf{I}(\boldsymbol{\theta})=$

$$
\left[\begin{array}{ccccc}
\mathrm{E}\left(-\frac{\partial^{2} \ell(\boldsymbol{\theta})}{\partial \alpha_{1}^{2}}\right) & \mathrm{E}\left(-\frac{\partial^{2} \ell(\boldsymbol{\theta})}{\partial \alpha_{1} \partial \beta_{1}}\right) & 0 & 0 & 0 \\
\mathrm{E}\left(-\frac{\partial^{2} \ell(\boldsymbol{\theta})}{\partial \beta_{1} \partial \alpha_{1}}\right) & \mathrm{E}\left(-\frac{\partial^{2} \ell(\boldsymbol{\theta})}{\partial \beta_{1}^{2}}\right) & 0 & 0 & 0 \\
0 & 0 & \mathrm{E}\left(-\frac{\partial^{2} \ell(\boldsymbol{\theta})}{\partial \sigma^{2}}\right) & 0 & 0 \\
0 & 0 & \mathrm{E}\left(-\frac{\partial^{2} \ell(\boldsymbol{\theta})}{\partial \alpha_{2}^{2}}\right) & \mathrm{E}\left(-\frac{\partial^{2} \ell(\boldsymbol{\theta})}{\partial \alpha_{2} \partial \beta_{2}}\right) \\
0 & 0 & \mathrm{E}\left(-\frac{\partial^{2} \ell(\boldsymbol{\theta})}{\partial \beta_{2} \partial \alpha_{2}}\right) & \mathrm{E}\left(-\frac{\partial^{2} \ell(\boldsymbol{\theta})}{\partial \beta_{2}^{2}}\right)
\end{array}\right]
$$


For the term $\mathrm{E}\left(-\partial^{2} \ell(\boldsymbol{\theta}) / \partial \alpha_{1}^{2}\right)$, after trivial derivations we have the expression of $-\partial^{2} \ell(\boldsymbol{\theta}) / \partial \alpha_{1}^{2}$ as follows:

$$
\begin{aligned}
-\frac{\partial^{2} \ell(\boldsymbol{\theta})}{\partial \alpha_{1}^{2}}=\sum_{j=1}^{J} & \sum_{i=1}^{N_{j}}\left[1_{\left\{2 \leq \delta_{i j} \leq K\right\}} \sum_{k=1}^{\delta_{i j}-1} \frac{2 \eta^{2}\left(x_{j}\right) \Delta t-y_{i j k} \eta\left(x_{j}\right)}{\sigma^{2}}\right. \\
& \left.+1_{\left\{\delta_{i j}=K+1\right\}} \sum_{k=1}^{K} \frac{2 \eta^{2}\left(x_{j}\right) \Delta t-y_{i j k} \eta\left(x_{j}\right)}{\sigma^{2}}\right]
\end{aligned}
$$

The expectation can be calculated by

$$
\begin{gathered}
\mathrm{E}\left(-\frac{\partial^{2} \ell(\boldsymbol{\theta})}{\partial \alpha_{1}^{2}}\right)=\sum_{j=1}^{J} \sum_{i=1}^{N_{j}} \mathrm{E}_{\delta_{i j}}\left[\mathrm { E } \left[1_{\left\{2 \leq \delta_{i j} \leq K\right\}} \sum_{k=1}^{\delta_{i j}-1} \frac{2 \eta^{2}\left(x_{j}\right) \Delta t-y_{i j k} \eta\left(x_{j}\right)}{\sigma^{2}}\right.\right. \\
\left.\left.+1_{\left\{\delta_{i j}=K+1\right\}} \sum_{k=1}^{K} \frac{2 \eta^{2}\left(x_{j}\right) \Delta t-y_{i j k} \eta\left(x_{j}\right)}{\sigma^{2}}\right]\right] \\
=\sum_{j=1}^{J} \sum_{i=1}^{N_{j}}\left[\sum_{l=2}^{K} \operatorname{Pr}\left(\delta_{i j}=l\right) \mathrm{E}\left(\sum_{k=1}^{l-1} \frac{2 \eta^{2}\left(x_{j}\right) \Delta t-y_{i j k} \eta\left(x_{j}\right)}{\sigma^{2}}\right)\right. \\
\left.\quad+\operatorname{Pr}\left(\delta_{i j}=K+1\right) \mathrm{E}\left(\sum_{k=1}^{K} \frac{2 \eta^{2}\left(x_{j}\right) \Delta t-y_{i j k} \eta\left(x_{j}\right)}{\sigma^{2}}\right)\right] \\
=\sum_{j=1}^{J} \sum_{i=1}^{N_{j}}\left[\sum_{l=2}^{K} \operatorname{Pr}\left(\delta_{i j}=l\right)(l-1) \frac{\eta^{2}\left(x_{j}\right) \Delta t}{\sigma^{2}}+\operatorname{Pr}\left(\delta_{i j}=K+1\right) K \frac{\eta^{2}\left(x_{j}\right) \Delta t}{\sigma^{2}}\right]
\end{gathered}
$$

As for $l=2, \ldots, K, \operatorname{Pr}\left(\delta_{i j}=l\right)=\exp \left[-\lambda\left(x_{j}\right)(l-1) \Delta t\right]-\exp \left[-\lambda\left(x_{j}\right) l \Delta t\right]$ and also $\operatorname{Pr}\left(\delta_{i j}=K+1\right)=\exp \left[-\lambda\left(x_{j}\right) K \Delta t\right]$, thus following Eq. (16) we have,

$$
\begin{aligned}
\mathrm{E}\left(-\frac{\partial^{2} \ell(\boldsymbol{\theta})}{\partial \alpha_{1}^{2}}\right) & =\sum_{j=1}^{J} \sum_{i=1}^{N_{j}}\left\{\sum_{l=2}^{K}\left\{\exp \left[-\lambda\left(x_{j}\right)(l-1) \Delta t\right]-\exp \left[-\lambda\left(x_{j}\right) l \Delta t\right]\right\}(l\right. \\
& \left.-1) \frac{\eta^{2}\left(x_{j}\right) \Delta t}{\sigma^{2}}+\exp \left[-\lambda\left(x_{j}\right) K \Delta t\right] K \frac{\eta^{2}\left(x_{j}\right) \Delta t}{\sigma^{2}}\right\}=\sum_{j=1}^{J} N_{j} \mathcal{F}_{j}
\end{aligned}
$$

Similarly, the other elements in $\mathbf{I}(\boldsymbol{\theta})$ are given. The details are given in Appendix A.

\subsection{Optimization problem formulation}

In this subsection, we formulate optimization problems for the three criteria. With the Fisher information given analytically, optimization based on [C1] and [C1] can be easily 
implemented. However, for [C3], we need to derive the asymptotic variance of the estimator of a certain lifetime percentile. To approximately evaluate the asymptotic variance by the Fisher information, we employ the delta method. Based on Eq. (5) and (6), the expression for $\operatorname{AVar}\left(\hat{t}_{p}\right)$ is given by

$$
\operatorname{AVar}\left(\hat{t}_{p}\right)=\frac{\boldsymbol{H}(\boldsymbol{\theta})[\mathbf{I}(\boldsymbol{\theta})]^{-1} \boldsymbol{H}(\boldsymbol{\theta})^{\prime}}{\left[f_{T}\left(t_{p}\right)\right]^{2}}
$$

where $\boldsymbol{H}(\boldsymbol{\theta})$ is the first derivative of $F_{T}\left(t_{p}\right)$ with respect to $\boldsymbol{\theta}$, i.e.,

$$
\boldsymbol{H}(\boldsymbol{\theta})=\left(\frac{\partial F_{T}\left(t_{p}\right)}{\partial \alpha_{1}}, \frac{\partial F_{T}\left(t_{p}\right)}{\partial \beta_{1}}, \frac{\partial F_{T}\left(t_{p}\right)}{\partial \sigma}, \frac{\partial F_{T}\left(t_{p}\right)}{\partial \alpha_{2}}, \frac{\partial F_{T}\left(t_{p}\right)}{\partial \beta_{2}}\right)
$$

The detailed expression of $\boldsymbol{H}(\boldsymbol{\theta})$ is given in Appendix B.

A test plan $\mathcal{P}$ includes the setting of $x_{i}$ and $\pi_{i}$, that is, $\mathcal{P}=\left(x_{1}, \ldots, x_{J}, \pi_{1}, \ldots, \pi_{J}\right)$. In general, the optimization problem is formulated as follows,

$$
\begin{array}{ll}
\text { Minimize } & -\operatorname{det}(\mathbf{I}(\boldsymbol{\theta})) \quad \text { for [C1] } \\
& \operatorname{tr}\left(\mathbf{I}^{-1}(\boldsymbol{\theta})\right) \quad \text { for [C2] } \\
& \operatorname{A} \operatorname{Var}\left(\hat{t}_{p}\right) \quad \text { for [C3] } \\
\text { subject to } \quad & 0 \leq x_{j} \leq 1, j=1, \ldots, J \\
& \sum_{j-1}^{J} \pi_{j}=1 \\
& \pi_{j} N=N_{j} \\
& N_{j} \in \mathbb{N}^{+}, j=1, \ldots, J
\end{array}
$$

\subsection{General equivalence theorems to verify global optimality}

For a test with $J$ stress levels, we have $2 J-1$ continuous variables between 0 and 1 to be optimized. As the objective function for each criterion is apparently nonlinear and difficult to evaluate the convexity, we have to resort to numerical optimization methods to solve the problem, which makes it important to verify the optimality of ADT plans. We use the Whittle's general equivalence theorems (GET) [31] to verify the global optimality of the plans. 
The GET is commonly used to verify the global optimality of a given experimental design derived by the Fisher information matrix or its associated functions. Following the previous GET literature, we define the derivative function at a test plan $\mathcal{P}$ and stress level $x$ as $d(\mathcal{P}, x)$ under different criteria as,

1. [C1]: D-optimality:

$$
d(\mathcal{P}, x)=\operatorname{tr}\left((\mathbf{I}(\boldsymbol{\theta} ; \mathcal{P}))^{-1} \mathbf{I}\left(\boldsymbol{\theta} ; \mathcal{P}_{x}\right)\right)-N_{\operatorname{dim}}
$$

where $\mathcal{P}_{x}$ is the 1-level plan in which all the test units are allocated to stress level $x$, $N_{\text {dim }}$ is the number of unknown parameters, i.e., the dimension of the Fisher information. In our case, $N_{\text {dim }}=5$.

2. [C2]: A-optimality [32]:

$$
d(\mathcal{P}, x)=\operatorname{tr}\left((\mathbf{I}(\boldsymbol{\theta} ; \mathcal{P}))^{-1} \mathbf{I}\left(\boldsymbol{\theta} ; \mathcal{P}_{x}\right)(\mathbf{I}(\boldsymbol{\theta} ; \mathcal{P}))^{-1}\right)-\operatorname{tr}\left((\mathbf{I}(\boldsymbol{\theta} ; \mathcal{P}))^{-1}\right)
$$

3. [C3]: $V$-optimality:

$$
d(\mathcal{P}, x)=\frac{\boldsymbol{H}(\boldsymbol{\theta})[\mathbf{I}(\boldsymbol{\theta} ; \mathcal{P})]^{-1} \mathbf{I}\left(\boldsymbol{\theta} ; \mathcal{P}_{x}\right)[\mathbf{I}(\boldsymbol{\theta} ; \mathcal{P})]^{-1} \boldsymbol{H}^{\prime}(\boldsymbol{\theta})}{\left[f_{T}\left(t_{p}\right)\right]^{2}}-\operatorname{AVar}\left(\hat{\xi}_{p} ; \boldsymbol{P}\right)
$$

According to the GET theorem, $\mathcal{P}^{*}$ is globally optimal if and only if $\sup _{x \in[0,1]} d\left(\mathcal{P}^{*}, x\right)=0$. To verify the optimality of $\mathcal{P}^{*}$, it is sufficient to show that $d\left(\mathcal{P}^{*}, x\right)=0$ for all $x$ belonging to $\mathcal{P}^{*}$ and $d\left(\mathcal{P}^{*}, x\right)<0$ otherwise. Therefore, plots of the derivative function against $x$ can be used to verify optimality.

\section{An application example}

\subsection{Optimal ADT planning to test carbon-film resistor}

The example of carbon-film resistor [7] is modified and revisited to illustrate the proposed methods. The degradation characteristic of interest is the percentage increase in the resistance of the resistor. It is assumed that at the beginning of the test the resistance is zero and the degradation process is well modeled by a linear Wiener process, denoted by $Y(t)$. The dominating factor that influences the degradation path was temperature, and higher temperature was proved to accelerate the degradation rate. The Arrhenius model is used to link the stress with model parameters. The maximum thermal environment that the test chamber can provide is 
$173{ }^{\circ} \mathrm{C}$ and the temperature under use condition is $50{ }^{\circ} \mathrm{C}$. The resistor is considered to fail when there is a $5 \%$ increase in resistance, i.e., $D=5$. We assume that there are 200 test units for the experiment and the maximum test duration is 1500 hours with the inspection interval 75 hours, i.e., $K=20, \Delta t=75$.

The stress variable is standardized based on the Arrhenius relationship; denote $T_{H}$ and $T_{0}$ as the maximum and use temperature in the Kelvin scale, respectively. For this example, $T_{H}=$ $446 \mathrm{~K}, T_{0}=323 \mathrm{~K}$. Denote $T_{j}$ as the temperature of the $j$-th stress, the standardized temperature $x$ is obtained as

$$
x_{j}=\frac{1 / T_{0}-1 / T_{l}}{1 / T_{0}-1 / T_{H}}=3.6260-\frac{1171}{T_{l}}
$$

which satisfies $x_{H}=1, x_{0}=0$.

Based on the pre-estimation and experts' opinions, the pilot parameters for the degradation acceleration model is set as $\left(\alpha_{1}, \beta_{1}, \sigma\right)=(-8.3,2.5,0.027)$. For the traumatic shocks, we assume that at the use condition, only $0.5 \%$ of the test units are expected to fail within the test duration $1500 \mathrm{hrs}$, and $30 \%$ of the test units fail under $173^{\circ} \mathrm{C}$. Therefore, the additional pilot setting is $\left(\alpha_{2}, \beta_{2}\right)=(-12.61,4.26)$, making $\boldsymbol{\theta}=(-8.3,2.5,0.027,-12.61,4.26)^{\prime}$. Figure 2 shows an illustration of the behaviors of 20 simulated test units under the maximum stress levels. As we can see there are 5 shock failures among the test units until the end of the test, and the degradation levels after the shocks are not observable. 


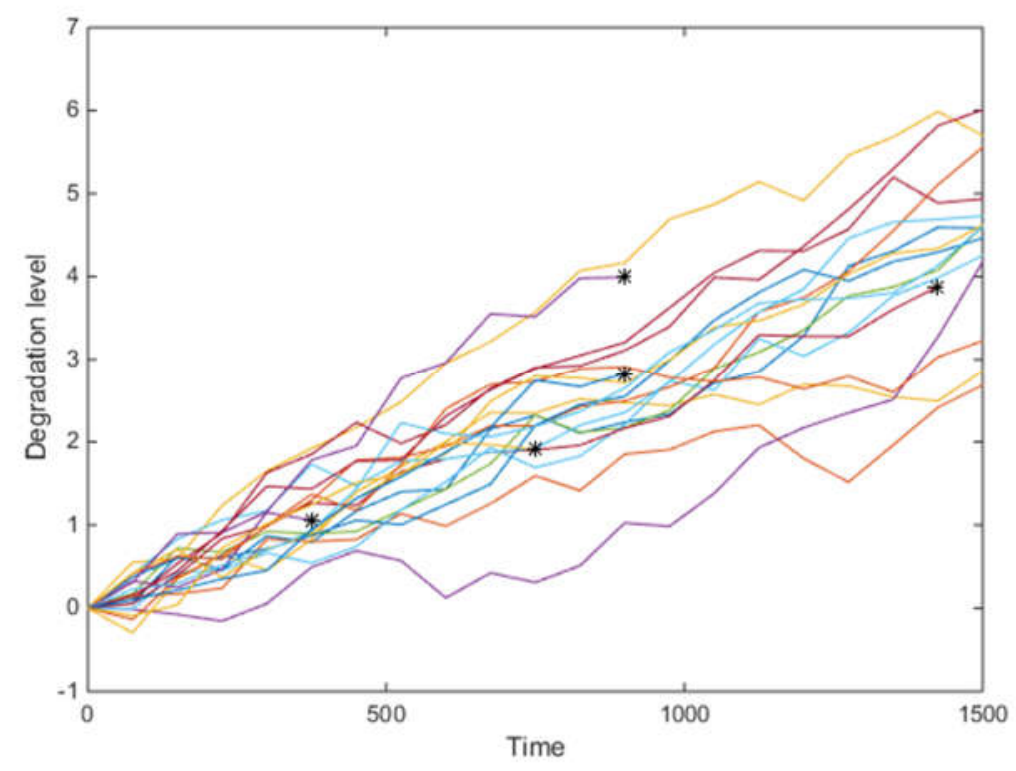

Figure 2 An illustration of the ADT test from a sample of 20 under the maximum stress. Asterisks (*) corresponds shock failures.

For [C3], we set $p=0.1$, that is, to minimize the asymptotic variance of estimated 0.1 quantile of the lifetime. The objective values under various plans under [C1], [C2] and [C3] are plotted in contour plots in Figure 3. By solving the optimization problems in Eq. (19), the twolevel optimal ADT plans are given in Table 1. For comparison, Table 2 lists the optimal ADT plans regardless of shock failures.
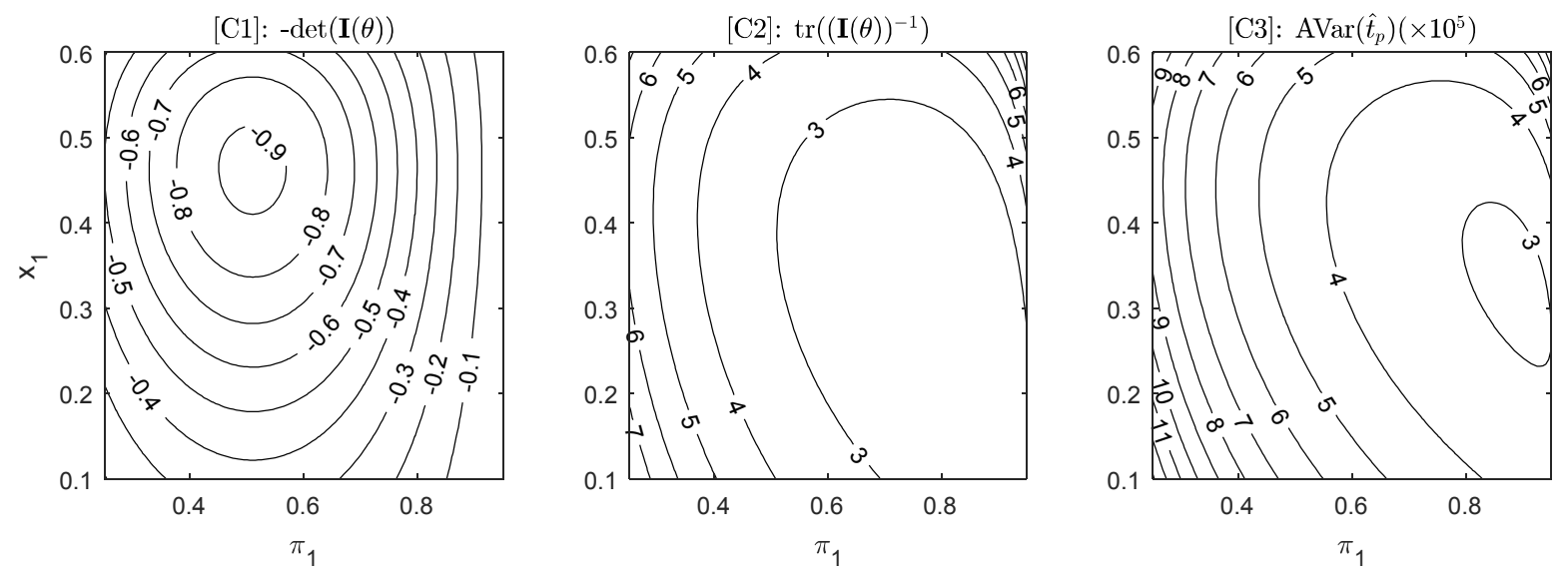

Figure 3 Contour plot of objective value under different $x_{1}$ and $\pi_{1}$ for [C1]-[C3] 
The contour plots intuitively show there is a unique local optimal plan that is also global optimal under each criterion. The optimal plan under [C1] is more sensitive to the change in $\pi_{1}$, while the optimal plan under [C2] or [C3] is more sensitive to the change in $x_{1}$.

Table 1 Optimal 2-level ADT plans

\begin{tabular}{|c|c|c|c|c|c|}
\hline \multirow[b]{2}{*}{ Criterion } & \multicolumn{4}{|c|}{ Optimal 2-level plan } & \multirow{2}{*}{$\begin{array}{c}\text { Objective } \\
\text { value }\end{array}$} \\
\hline & & $x_{j}$ & $\operatorname{temp}^{\circ} \mathrm{C}$ & $\pi_{j}$ & \\
\hline \multirow{2}{*}[\mathrm{C}1]{} & Low & 0.553 & 108.0 & 0.510 & \multirow{2}{*}{$\operatorname{det}(\mathbf{I}(\boldsymbol{\theta}))=9.27 \times 10^{12}$} \\
\hline & High & 1 & 173.0 & 0.490 & \\
\hline \multirow{2}{*}[\mathrm{C}2]{} & Low & 0.397 & 89.6 .0 & 0.815 & \multirow{2}{*}{$\operatorname{tr}\left(\mathbf{I}^{-1}(\boldsymbol{\theta})\right)=2.25$} \\
\hline & High & 1 & 173.0 & 0.185 & \\
\hline \multirow{2}{*}{ [C3] } & Low & 0.422 & 92.5 & 0.890 & \multirow{2}{*}{$\mathrm{A} \operatorname{Var}\left(\hat{t}_{0.1}\right)=2.87 \times 10^{5}$} \\
\hline & High & 1 & 173.0 & 0.110 & \\
\hline
\end{tabular}

Table 2 Optimal 2-level ADT plans with only degradation failures

\begin{tabular}{|c|c|c|c|c|c|}
\hline \multirow[b]{2}{*}{ Criterion } & \multicolumn{4}{|c|}{ with only degradation failures } & \multirow{2}{*}{$\begin{array}{c}\text { Objective } \\
\text { value }\end{array}$} \\
\hline & & $x_{j}$ & temp ${ }^{\circ} \mathrm{C}$ & $\pi_{j}$ & \\
\hline \multirow{2}{*}[\mathrm{C}1]{} & Low & 0.600 & 114.0 & 0.500 & \multirow{2}{*}{$\operatorname{det}(\mathbf{I}(\boldsymbol{\theta}))=8.45 \times 10^{11}$} \\
\hline & High & 1 & 173.0 & 0.500 & \\
\hline \multirow{2}{*}{ [C2] } & Low & 0.493 & 100.8 & 0.820 & \multirow{2}{*}{$\operatorname{tr}\left(\mathbf{I}^{-1}(\boldsymbol{\theta})\right)=0.039$} \\
\hline & High & 1 & 173.0 & 0.180 & \\
\hline \multirow{2}{*}{ [C3] } & Low & 0.489 & 100.3 & 0.880 & \multirow{2}{*}{$\mathrm{A} \operatorname{Var}\left(\hat{t}_{0.1}\right)=1.54 \times 10^{5}$} \\
\hline & High & 1 & 173.0 & 0.120 & \\
\hline
\end{tabular}

For all the three criteria, the optimal ADT plans with shock consideration have smaller lower stress level and more units allocated at lower stress compared with those regardless of shock 
failures. This is because the presence of shock failures prevents the optimal plan from exploring more at the maximum stress due to the risk of information loss caused by shocks. In both cases, by comparison, for [C1] the optimal plans tend to allocate more test units at higher stress levels, while for [C2] and [C3] the optimal plans allocate significantly more units at the lower stress. It is interesting to observe that for [C2], the optimal lower stress in Table 1 is significantly smaller than Table 2, and the objective value also differs drastically. The reason behind this is that by considering shock failures, the variability of the two additional parameters contributes significantly to the sum of variance of all parameters because the information on shock failures is much less than degradation failures. Therefore, the optimization under [C2] is dominated by the shock model. It is also noted that even though there are considerable differences in optimal stress levels from Table 1 and Table 2, there is only very slight difference in optimal proportions.

As advised in Section 3.3, we should verify the global optimality of the ALT plans given in Table 1. By utilizing the GET, we plot the derivative functions against the lower stress level $x_{1}$ under three criteria in Figure 4. The figure shows that the 2-level plans given in Table 1 are the globally optimal plans over the feasible temperature range in the experiment. It is noted that the GET ensures that the plans we obtained are optimal, but cannot ensure the uniqueness of these optimal plans. Nevertheless, the contour plots in Section 4.1 have supported that the optimal plans are unique within the feasible range of decision variables.
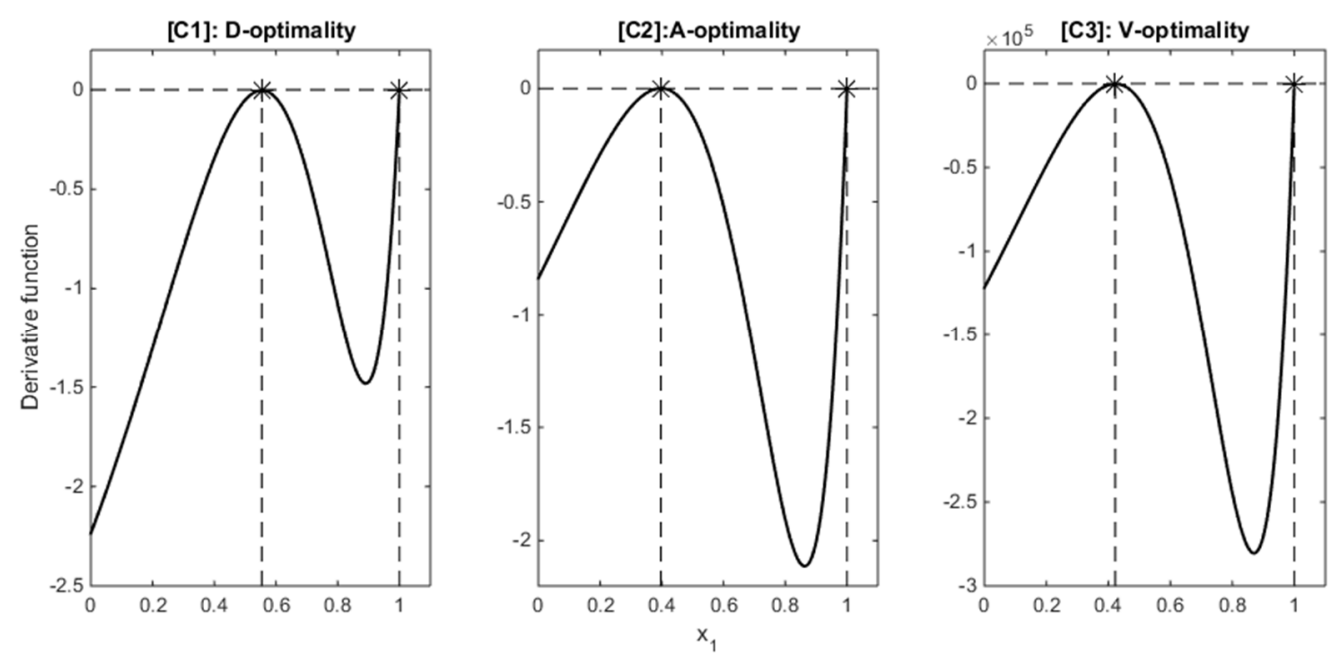

Figure 4 Derivative function against lower stress level $x_{1}$ under [C1], [C2], [C3] 


\subsection{Simulation study}

To investigate the possible outcomes of the experiment, we use Monte Carlo simulation to illustrate the sampling variability by assuming that the pilot parameters are true. A total of 1000 sets of ADT data were generated under optimal plans, and we calculate the MLE for unknown parameters based on each sample. The average and standard deviation for each MLE under [C1], [C2] and [C3] are shown in Table 3. We can see that the simulated outcomes are quite close to the theoretical results. Moreover, under the current optimal plan setting for the pilot parameters, the precision of the estimated parameters is high. Therefore, the optimal plans should yield reasonable efficiency in most cases if the pilot parameters are reasonable.

Table 3 A simulation study of optimal plans

\begin{tabular}{|c|c|c|c|c|}
\hline & \multicolumn{3}{|c|}{ Criterion } & \multirow{3}{*}{$\begin{array}{l}\text { True } \\
\text { value }\end{array}$} \\
\hline & {$[\mathrm{C} 1]$} & {$[\mathrm{C} 2]$} & [C3] & \\
\hline $\mathcal{P}^{*}$ & $(0.553,1,0.51,0.49)$ & $(0.397,1,0.82,0.19)$ & $(0.422,1,0.89,0.11)$ & \\
\hline $\operatorname{Avg}\left(\hat{\alpha}_{1}\right)$ & -8.3112 & -8.3107 & -8.2999 & -8.3 \\
\hline $\operatorname{sd}\left(\hat{\alpha}_{1}\right)$ & 0.1599 & 0.1457 & 0.1317 & \\
\hline $\operatorname{Avg}\left(\hat{\beta}_{1}\right)$ & 2.5096 & 2.5082 & 2.4975 & 2.5 \\
\hline $\operatorname{sd}\left(\hat{\beta}_{1}\right)$ & 0.1685 & 0.1605 & 0.1607 & \\
\hline $\operatorname{Avg}(\hat{\sigma})$ & 0.0270 & 0.0270 & 0.0270 & 0.027 \\
\hline $\operatorname{Std}(\hat{\sigma})$ & 0.0003 & 0.0003 & 0.0003 & \\
\hline $\operatorname{Avg}\left(\hat{\alpha}_{2}\right)$ & -12.8589 & -12.8594 & -12.7381 & -12.61 \\
\hline $\operatorname{sd}\left(\hat{\alpha}_{2}\right)$ & 1.1581 & 1.0092 & 0.8044 & \\
\hline $\operatorname{Avg}\left(\hat{\alpha}_{2}\right)$ & 4.5037 & 4.4774 & 4.3320 & 4.27 \\
\hline $\operatorname{sd}\left(\hat{\alpha}_{2}\right)$ & 1.2116 & 1.1209 & 1.1442 & \\
\hline Obj. value & $1.28 \times 10^{13}$ & 2.322 & $2.91 \times 10^{5}$ & \\
\hline Theoretical & $9.27 \times 10^{12}$ & 2.245 & $2.87 \times 10^{5}$ & \\
\hline Obj. value & & & & \\
\hline
\end{tabular}

\subsection{3-level compromise plans}

In Section 4.1, we have demonstrated that the 2-level test plans are optimal under the three criteria of interest. However, in practice, the 3-level compromise plans may be adopted to check 
the validity of the acceleration regression model, especially in case of significant nonlinearity in the model in the experimental region. For the compromise plans, an extra stress level $x_{m}$ is added to experiment, where $x_{m}=\left(x_{1}^{*}+x_{2}^{*}\right) / 2$. As in Tseng and Lee [33] three common rules for ADT compromise plans are as follows:

- $\mathbf{R}_{1}$ : The proportion of allocation is fixed at $4: 2: 1$. The stress levels need to be optimized.

- $\mathbf{R}_{2}$ : The proportion of allocation of the added stress level is fixed at $10 \%$. Other variables of the plan need to be optimized.

- $\mathbf{R}_{3}$ : The proportion of allocation of the added stress level is fixed at $20 \%$. Other variables of the plan need to be optimized.

The relative efficiency (RE) for each optimal compromise 3-level plan $\mathcal{C} \mathcal{P}_{i}^{*}$ with respect to the 2-level globally optimal plan $\mathcal{P}_{i}^{*}$ for $i=D, A, V$ representing the three optimality criteria is given as follows,

- $\quad[\mathrm{C} 1]: \operatorname{RE}=\operatorname{det}\left(\mathbf{I}\left(\boldsymbol{\theta} ; \boldsymbol{\mathcal { P }} \mathcal{P}_{i}^{*}\right)\right) / \operatorname{det}\left(\mathbf{I}\left(\boldsymbol{\theta} ; \mathcal{P}_{i}^{*}\right)\right)$;

- $\quad[\mathrm{C} 2]: \operatorname{RE}=\operatorname{tr}\left(\mathbf{I}^{-1}\left(\boldsymbol{\theta} ; \mathcal{P}_{i}^{*}\right)\right) / \operatorname{tr}\left(\mathbf{I}^{-1}\left(\boldsymbol{\theta} ; \boldsymbol{\mathcal { P }} \mathcal{P}_{i}^{*}\right)\right)$;

- $\quad[\mathrm{C} 3]: \mathrm{RE}=\mathrm{AVar}\left(\hat{t}_{p} ; \mathcal{P}_{i}^{*}\right) / \mathrm{A} \operatorname{Var}\left(\hat{t}_{p} ; \mathcal{C} \mathcal{P}_{i}^{*}\right)$.

Based on the three planning rules, we derive the optimal compromise plans and compute the RE under each criterion respectively. The results are summarized in Table 4.

Table 4 Optimal compromise ADT plans under [C1], [C2] and [C3]

\begin{tabular}{|c|c|c|c|c|c|c|}
\hline \multirow{2}{*}{ Criterion } & \multirow{2}{*}{ Rules } & \multicolumn{4}{|c|}{ Optimal 3-level compromise plan } & \multirow{2}{*}{$\mathrm{RE}$} \\
\hline & & & $x_{i}$ & temp $^{\circ} \mathrm{C}$ & $\pi_{j}$ & \\
\hline \multirow{9}{*}[\mathrm{C}1]{} & \multirow{3}{*}{$\mathbf{R}_{1}$} & Low & 0.52 & 104.0 & 0.57 & \multirow{3}{*}{0.270} \\
\hline & & Middle & 0.76 & 135.6 & 0.29 & \\
\hline & & High & 1.00 & 173.0 & 0.15 & \\
\hline & \multirow{3}{*}{$\mathbf{R}_{2}$} & Low & 0.52 & 104.0 & 0.43 & \multirow{3}{*}{0.914} \\
\hline & & Middle & 0.76 & 135.6 & 0.10 & \\
\hline & & High & 1.00 & 173.0 & 0.47 & \\
\hline & \multirow{3}{*}{$\mathbf{R}_{3}$} & Low & 0.48 & 99.2 & 0.34 & \multirow{3}{*}{0.844} \\
\hline & & Middle & 0.74 & 132.8 & 0.20 & \\
\hline & & High & 1.00 & 173.0 & 0.47 & \\
\hline$[\mathrm{C} 2]$ & & Low & 0.26 & 74.9 & 0.57 & 0.867 \\
\hline
\end{tabular}




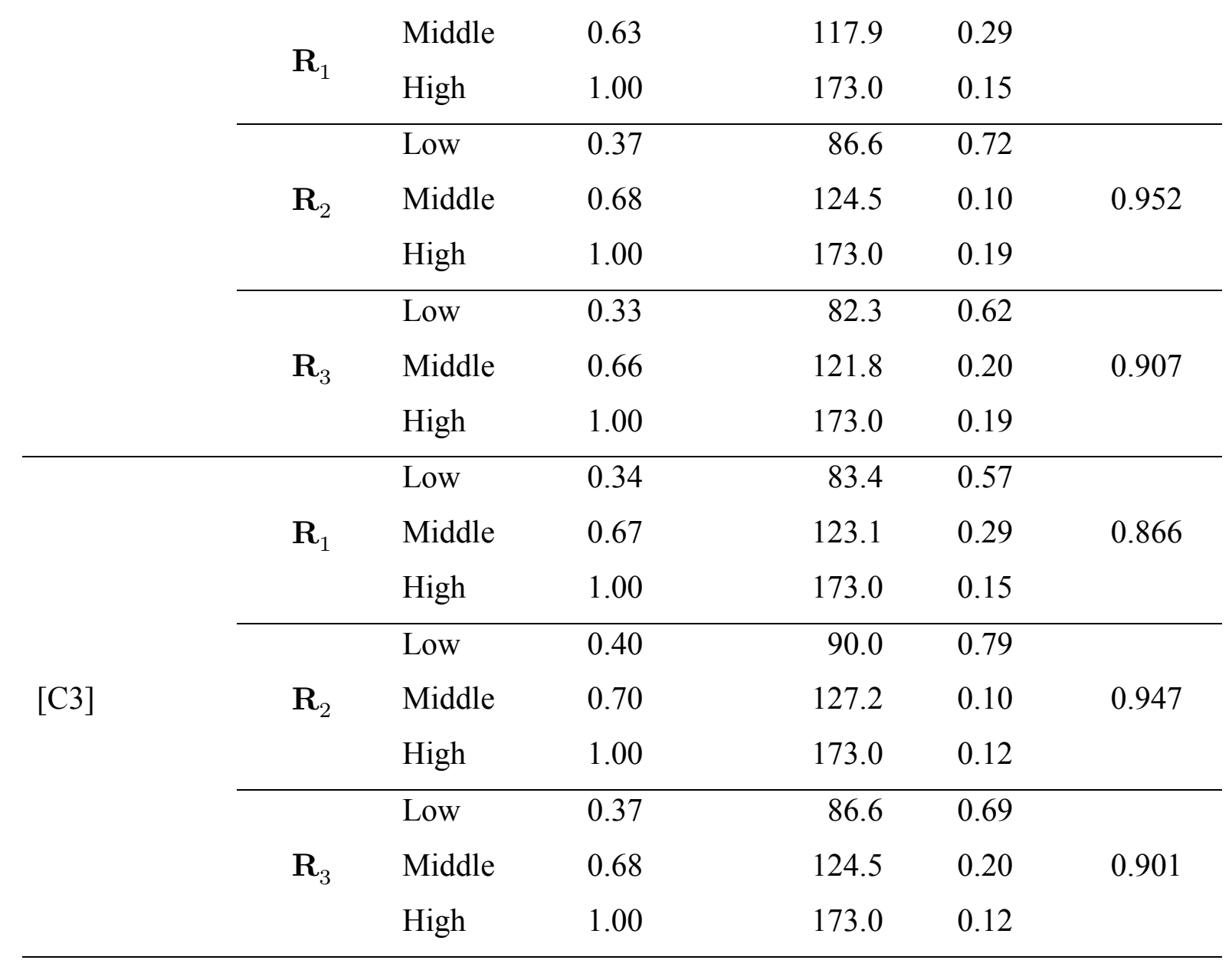

The REs shown in the table demonstrate that the performance of $\mathbf{R}_{2}$ and $\mathbf{R}_{3}$ is better than $\mathbf{R}_{1}$ under all the criteria. Nevertheless, $\mathbf{R}_{1}$ has reasonable REs that is over $85 \%$ under [C2] and [C3], but the RE of $\mathbf{R}_{1}$ is extremely low under [C1] because the 4:2:1 allocation rule is far from optimal allocations under [C1]. It is straightforward that the performance of $\mathbf{R}_{2}$ is better than $\mathbf{R}_{3}$ as it allocates less test units under the compromise additional stress level. However, this also weakens the statistical capability to validate the model, which is the main objective of the compromise. In general, $\mathbf{R}_{2}$ or $\mathbf{R}_{3}$ are recommended as the rules for compromise plans, based on the test planners' confidence in the pilot acceleration regression model and the number of available test units. With the compromise plans, degradation and lifetime data from three stress levels are obtained in a test. Three-level data provide possibility to verify the acceleration regression models in Eqs. (7)-(8). For example, if the true model in Eq. (7) is a log-quadratic model rather than log-linear, i.e., $\eta(x)=\exp \left(\alpha_{1}+\beta_{1} x+\gamma_{1} x^{2}\right)$, we can fit the three-level data to the alternative model to verify if it is more appropriate, and it is noted that it is not applicable for two-level data. In practice, the compromise plans are suggested to be used if the prior information of the acceleration model is not believed to be very reliable [34], [35, p. 341]. 


\section{Sensitivity analysis and robustness of the test plan}

As the robustness is of great importance in application of a test plan, in this section, we investigate the effects of various changes in test planning inputs on the optimal plans to explore the robustness of the proposed plans.

\subsection{A general sensitivity analysis}

The pilot model parameters are usually given by experts or preliminary estimation; thus they are subject to estimation errors. To study the influence of estimation errors on the optimal plans, we increase and decrease each parameter by $10 \%$ and obtain the optimal plans. We use a Taguchi orthogonal design $L_{27}$ with 5 factors (parameters) and 3 levels, where +1 and -1 represents $10 \%$ increase and decrease in the pilot parameter and 0 stands for no change. The optimal plans under each setting are given in Table 5. A summary is made to show the range and standard deviation of each optimal plan setting by varying the parameters under each criterion in Table 6. We notice that all the optimal plans have the high stress level at 1 and the variation of allocation proportions is slight, thus we concentrate on the changes in the optimal lower stress level $x_{1}^{*}$. Table 7 shows the effect rankings on $x_{1}$ for unknown parameters based on the analysis of the Taguchi design, and rank 1 represents the parameter with the largest effect.

Table 5 Effects of parameter changes on the optimal 2-level ADT plans

\begin{tabular}{|c|c|c|c|c|c|c|c|c|c|c|c|c|c|c|c|c|}
\hline \multirow[b]{3}{*}{$\epsilon_{1}$} & \multirow[b]{3}{*}{$\epsilon_{2}$} & \multirow[b]{3}{*}{$\epsilon_{3}$} & \multirow[b]{3}{*}{$\epsilon_{4}$} & & \multicolumn{12}{|c|}{ Optimal 2-level plan } \\
\hline & & & & & \multicolumn{4}{|c|}{$[\mathrm{C} 1]$} & \multicolumn{4}{|c|}{ [C2] } & \multicolumn{4}{|c|}{ [C3] } \\
\hline & & & & $\epsilon_{5}$ & $x_{1}$ & $x_{2}$ & $\pi_{1}$ & $\pi_{2}$ & $x_{1}$ & $x_{2}$ & $\pi_{1}$ & $\pi_{2}$ & $x_{1}$ & $x_{2}$ & $\pi_{1}$ & $\pi_{2}$ \\
\hline-1 & -1 & -1 & -1 & -1 & 0.48 & 1 & 0.52 & 0.48 & 0.31 & 1 & 0.81 & 0.19 & 0.31 & 1 & 0.92 & 0.09 \\
\hline-1 & -1 & -1 & -1 & 0 & 0.50 & 1 & 0.53 & 0.47 & 0.36 & 1 & 0.81 & 0.20 & 0.36 & 1 & 0.90 & 0.11 \\
\hline-1 & -1 & -1 & -1 & +1 & 0.50 & 1 & 0.55 & 0.46 & 0.40 & 1 & 0.79 & 0.22 & 0.38 & 1 & 0.89 & 0.12 \\
\hline-1 & 0 & 0 & 0 & -1 & 0.54 & 1 & 0.51 & 0.50 & 0.34 & 1 & 0.82 & 0.18 & 0.40 & 1 & 0.90 & 0.11 \\
\hline-1 & 0 & 0 & 0 & 0 & 0.55 & 1 & 0.51 & 0.49 & 0.40 & 1 & 0.82 & 0.19 & 0.43 & 1 & 0.89 & 0.11 \\
\hline-1 & 0 & 0 & 0 & +1 & 0.57 & 1 & 0.52 & 0.49 & 0.44 & 1 & 0.81 & 0.19 & 0.45 & 1 & 0.88 & 0.12 \\
\hline-1 & +1 & +1 & +1 & -1 & 0.57 & 1 & 0.50 & 0.50 & 0.34 & 1 & 0.83 & 0.18 & 0.49 & 1 & 0.88 & 0.13 \\
\hline-1 & +1 & +1 & +1 & 0 & 0.59 & 1 & 0.50 & 0.50 & 0.41 & 1 & 0.82 & 0.18 & 0.50 & 1 & 0.88 & 0.13 \\
\hline-1 & +1 & +1 & +1 & +1 & 0.60 & 1 & 0.51 & 0.50 & 0.46 & 1 & 0.82 & 0.19 & 0.51 & 1 & 0.88 & 0.13 \\
\hline 0 & -1 & 0 & +1 & -1 & 0.52 & 1 & 0.50 & 0.50 & 0.34 & 1 & 0.83 & 0.18 & 0.41 & 1 & 0.90 & 0.11 \\
\hline
\end{tabular}




\begin{tabular}{lllll|llll|llll|llll}
\hline 0 & -1 & 0 & +1 & 0 & 0.54 & 1 & 0.51 & 0.50 & 0.40 & 1 & 0.82 & 0.18 & 0.42 & 1 & 0.90 & 0.11 \\
0 & -1 & 0 & +1 & +1 & 0.56 & 1 & 0.51 & 0.50 & 0.46 & 1 & 0.82 & 0.19 & 0.43 & 1 & 0.89 & 0.11 \\
0 & 0 & +1 & -1 & -1 & 0.51 & 1 & 0.52 & 0.48 & 0.32 & 1 & 0.81 & 0.19 & 0.31 & 1 & 0.91 & 0.09 \\
0 & 0 & +1 & -1 & 0 & 0.52 & 1 & 0.53 & 0.47 & 0.37 & 1 & 0.80 & 0.20 & 0.36 & 1 & 0.90 & 0.11 \\
0 & 0 & +1 & -1 & +1 & 0.52 & 1 & 0.55 & 0.46 & 0.40 & 1 & 0.79 & 0.22 & 0.39 & 1 & 0.88 & 0.12 \\
0 & 1 & -1 & 0 & -1 & 0.56 & 1 & 0.51 & 0.50 & 0.34 & 1 & 0.82 & 0.18 & 0.39 & 1 & 0.90 & 0.11 \\
0 & 1 & -1 & 0 & 0 & 0.58 & 1 & 0.51 & 0.49 & 0.40 & 1 & 0.82 & 0.19 & 0.43 & 1 & 0.89 & 0.11 \\
0 & 1 & -1 & 0 & +1 & 0.59 & 1 & 0.52 & 0.49 & 0.44 & 1 & 0.81 & 0.19 & 0.46 & 1 & 0.88 & 0.12 \\
1 & -1 & +1 & 0 & -1 & 0.51 & 1 & 0.51 & 0.50 & 0.35 & 1 & 0.82 & 0.18 & 0.36 & 1 & 0.91 & 0.10 \\
1 & -1 & +1 & 0 & 0 & 0.53 & 1 & 0.51 & 0.49 & 0.40 & 1 & 0.82 & 0.19 & 0.40 & 1 & 0.90 & 0.11 \\
1 & -1 & +1 & 0 & +1 & 0.54 & 1 & 0.52 & 0.49 & 0.44 & 1 & 0.81 & 0.19 & 0.43 & 1 & 0.89 & 0.12 \\
1 & 0 & -1 & +1 & -1 & 0.54 & 1 & 0.50 & 0.50 & 0.34 & 1 & 0.83 & 0.18 & 0.43 & 1 & 0.89 & 0.11 \\
1 & 0 & -1 & +1 & 0 & 0.56 & 1 & 0.51 & 0.50 & 0.41 & 1 & 0.82 & 0.18 & 0.45 & 1 & 0.89 & 0.12 \\
1 & 0 & -1 & +1 & 1 & 0.58 & 1 & 0.51 & 0.50 & 0.46 & 1 & 0.82 & 0.19 & 0.47 & 1 & 0.88 & 0.12 \\
1 & +1 & 0 & -1 & -1 & 0.54 & 1 & 0.52 & 0.48 & 0.36 & 1 & 0.81 & 0.20 & 0.31 & 1 & 0.92 & 0.09 \\
1 & +1 & 0 & -1 & 0 & 0.54 & 1 & 0.53 & 0.47 & 0.40 & 1 & 0.80 & 0.20 & 0.36 & 1 & 0.90 & 0.11 \\
1 & +1 & 0 & -1 & +1 & 0.54 & 1 & 0.55 & 0.46 & 0.42 & 1 & 0.79 & 0.22 & 0.39 & 1 & 0.89 & 0.11 \\
\hline
\end{tabular}

Table 6 Summary of the general sensitivity analysis

\begin{tabular}{ccccc}
\hline \multirow{2}{*}{ Criterion } & \multicolumn{4}{c}{ Optimal 2-level plan } \\
\cline { 2 - 5 } & range $\left(x_{1}\right)$ & $\operatorname{range}\left(\pi_{1}\right)$ & $\operatorname{std}\left(x_{1}\right)$ & $\operatorname{std}\left(\pi_{1}\right)$ \\
\hline$[\mathrm{C} 1]$ & 0.1173 & 0.0450 & 0.0301 & 0.0142 \\
{$[\mathrm{C} 2]$} & 0.1440 & 0.0400 & 0.0436 & 0.0116 \\
{$[\mathrm{C} 3]$} & 0.2086 & 0.0400 & 0.0558 & 0.0111 \\
\hline
\end{tabular}

Table 7 Effects ranking of parameters on the optimal lower stress level

\begin{tabular}{cccccc}
\hline Criterion & \multicolumn{5}{c}{ Ranks of effect on $x_{1}^{*}$} \\
\cline { 2 - 6 } & 1 & 2 & 3 & 4 & 5 \\
\hline$[\mathrm{C} 1]$ & $\beta_{1}$ & $\alpha_{2}$ & $\beta_{2}$ & $\alpha_{1}$ & $\sigma$ \\
{$[\mathrm{C} 2]$} & $\beta_{2}$ & $\alpha_{2}$ & $\alpha_{1}$ & $\beta_{1}$ & $\sigma$ \\
{$[\mathrm{C} 3]$} & $\alpha_{2}$ & $\beta_{2}$ & $\beta_{1}$ & $\alpha_{1}$ & $\sigma$ \\
\hline
\end{tabular}


The results show that optimal plans under [C3] are more sensitive to the variation of parameters than those under [C1] and [C2]. Nevertheless, as Table 5 shows, the optimal plans do not change drastically when the parameters are changed in the range of $10 \%$ unless several parameters are overestimated or underestimated simultaneously, which rarely happens in practice because the correlations of the parameters are generally negative. Under the three criteria, the shock model parameters show at least equal significant effects on the optimal plans as the degradation parameters. For [C2] and [C3], the shock model parameters have larger effects on the optimal plans. It is noted that the intercept of shock acceleration model $\alpha_{2}$ ranks highest under [C3]. This is due to the fact that [C3] concerns more on the accuracy of extrapolation of the lifetime, and the intercept parameter of shock models determine the shock failure rate under use condition, which is of great importance when test planners try to predict the field lifetime. The degradation diffusion parameter $\sigma$ always exerts the least influence on the test planning.

\subsection{Effect of shock failure model}

The previous section has shown that the parameters on shock failures influence the optimal ADT plans in a more sensitive way, which motivates us to conduct a further sensitivity analysis on these parameters from a more intuitive perspective to the engineers. We change the proportions of shock failures under use and maximum stress levels to see how optimal plans vary under [C1], [C2] and [C3]. Note that these proportions are set by test planners prior to the experiment based on their experience and preliminary estimation, and the settings directly change the parameters $\alpha_{2}, \beta_{2}$ in the ADT model. The optimal plans are listed in Table 8-10 for [C1], [C2] and [C3], respectively. For convenience, we denote PF0 and PF1 as the proportions of shock failures use stress and maximum stress levels respectively.

Table 8 Optimal ADT plans under [C1] ( $D$-optimaility) under different shock failure assumptions.

\begin{tabular}{|c|c|c|c|c|c|c|c|}
\hline \multirow{6}{*}{ PF0 } & & & \multicolumn{5}{|c|}{ PF1 } \\
\hline & & & $10 \%$ & $30 \%$ & $50 \%$ & $70 \%$ & $90 \%$ \\
\hline & \multirow{2}{*}{$0.005 \%$} & $x_{j}$ & $0.68,1.00$ & $0.71,1.00$ & $0.71,1.00$ & $0.71,1.00$ & $0.72,1.00$ \\
\hline & & $\pi_{j}$ & $0.50,0.50$ & $0.51,0.49$ & $0.52,0.48$ & $0.53,0.47$ & $0.55,0.45$ \\
\hline & \multirow{2}{*}{$0.01 \%$} & $x_{j}$ & $0.66,1.00$ & $0.69,1.00$ & $0.70,1.00$ & $0.70,1.00$ & $0.70,1.00$ \\
\hline & & $\pi_{j}$ & $0.50,0.50$ & $0.51,0.49$ & $0.52,0.48$ & $0.53,0.47$ & $0.55,0.45$ \\
\hline
\end{tabular}




\begin{tabular}{rrrrrrr}
\hline $0.1 \%$ & $x_{j}$ & $0.58,1.00$ & $0.62,1.00$ & $0.64,1.00$ & $0.64,1.00$ & $0.64,1.00$ \\
& $\pi_{j}$ & $0.50,0.50$ & $0.51,0.49$ & $0.52,0.48$ & $0.53,0.47$ & $0.55,0.45$ \\
\hline \multirow{2}{*}{$1 \%$} & $x_{j}$ & $0.45,1.00$ & $0.51,1.00$ & $0.54,1.00$ & $0.55,1.00$ & $0.55,1.00$ \\
& $\pi_{j}$ & $0.50,0.50$ & $0.51,0.49$ & $0.52,0.48$ & $0.53,0.47$ & $0.55,0.45$
\end{tabular}

Table 9 Optimal ADT plans under [C2] ( $A$-optimaility) under different shock failure assumptions.

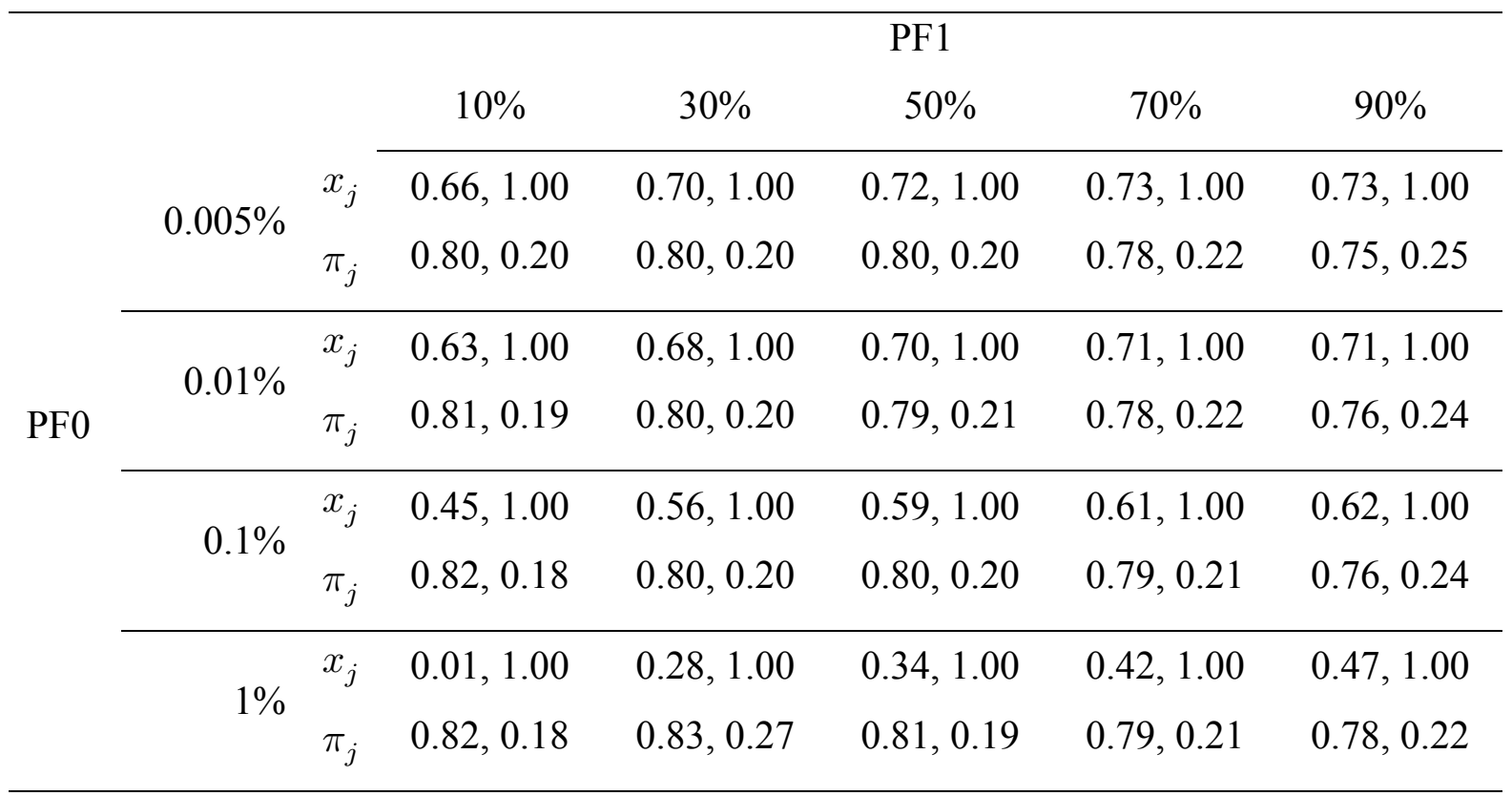

Table 10 Optimal ADT plans under [C3] ( $V$-optimaility) under different shock failure assumptions.

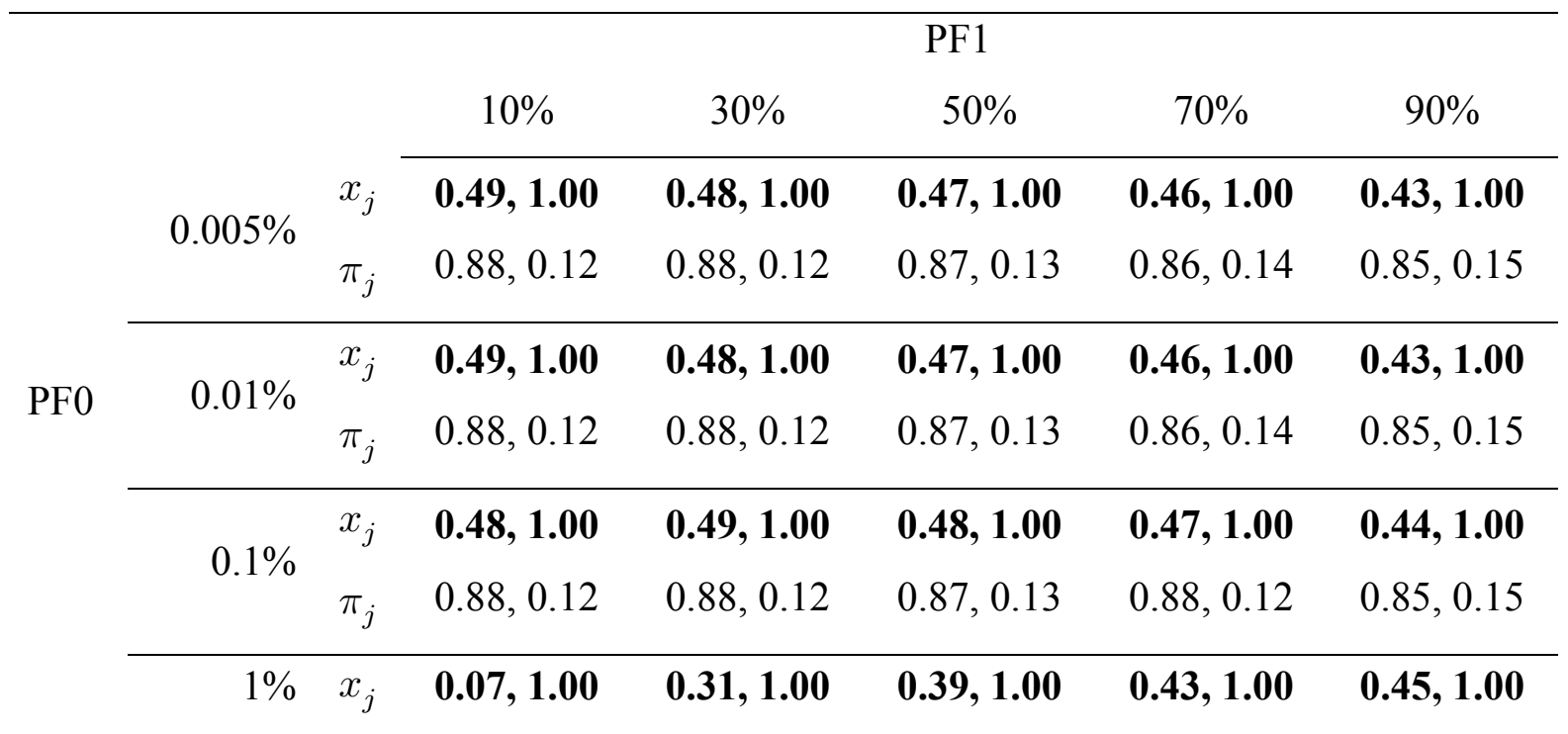




$\pi_{j} \quad 0.98,0.02 \quad 0.92,0.08 \quad 0.90,0.10 \quad 0.88,0.13 \quad 0.85,0.15$

Under all the three criteria, as PF0 increases, the optimal lower stress gets lower and the respective allocation proportion gets larger. However, we see some differences in the change of optimal plans under different criteria. For [C1] and [C2], as PF1 increases, the optimal lower stress becomes larger and fewer test units are allocated to it. On the contrary, as PF1 increases under [C3], the optimal lower stress becomes lower and fewer test units are allocated to it for $\mathrm{PH} 0$ from $0.005 \%$ to $0.1 \%$. For [C3], it is interesting to observe that when PF0 is large enough, such as $1 \%$, the optimal stress lower stress increases as PF1 increases. In addition, when PF0 and $\mathrm{PF} 1$ are relatively near, for example, when $\mathrm{PF} 0=1 \%$ and $\mathrm{PF} 1=10 \%$ or $30 \%$, almost all test units are allocated to a very low stress level. Under this assumption, because the shock failures occur very early under normal stress, thus a slightly elevated lower stress can produce a reasonable number of shock failures. Moreover, when PF0 and PF1 are both large, the shock failure parameters play a more significant role in predicting lifetime. This is also the reason that we have seen a considerable difference in optimal plans under $\mathrm{PF} 0=1 \%$ and other $\mathrm{PF} 0$ settings. In practice, the test planners should be careful when estimating the pilot parameters of shock failure models. If the shock intensity is underestimated, the derived ADT plan may deviate significantly from the true optimal plan. The result of such parameter misspecification may lead to a significant reduce of information from the real test.

\subsection{Effect of percentile of interest}

Although engineers or managers usually concern about a lower percentile of lifetime for a product to support decision making, the specific value of $p$ may vary due to different needs. Because $p$ does not influence the optimal plans under [C1] and [C2], we change $p$ from 0.1 to 0.9 to see the variation in optimal plans under [C3]. The optimal lower stress level and proportion is shown in Figure 5 and the optimal asymptotic variance of $\hat{t}_{p}$ is plotted in Figure 6. 


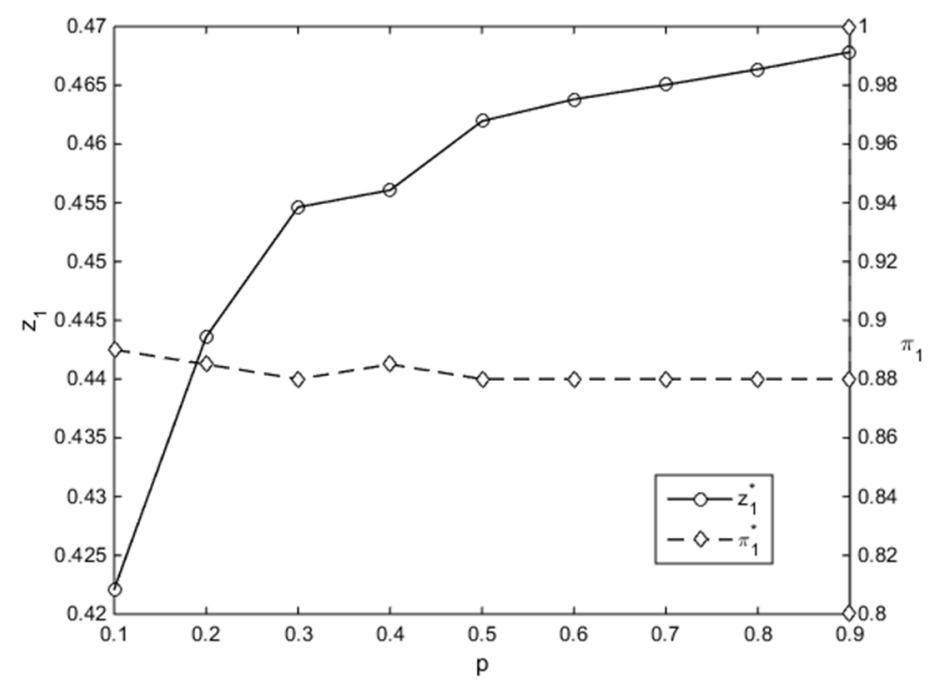

Figure 5 Optimal test plans under [C3] when $p$ varies

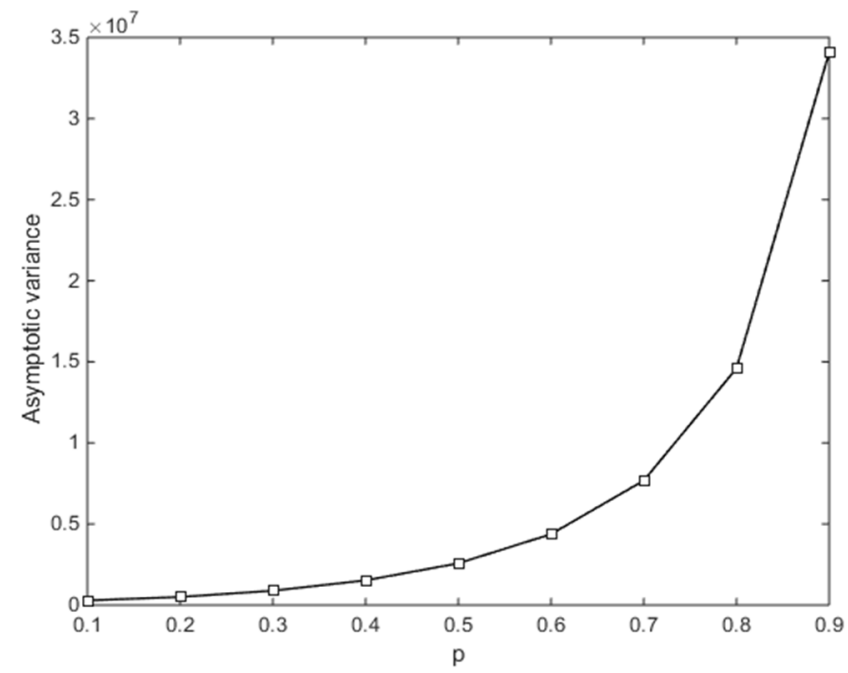

Figure 6 Minimized asymptotic variance of $\widehat{t_{p}}$ when $p$ varies

The optimal proportion allocated to lower stress almost remains unchanged when $p$ varies from 0.1 to 0.9 , and the optimal lower stress level increases from 0.42 to 0.47 . The reason of the increase in $x_{1}$ is that more failures need to be observed for inference of a higher percentiles. The minimum $\mathrm{AVar}\left(\hat{t}_{p}\right)$ increases drastically with $p$, but stays relatively small with a small $p$. The change in optimal plans with various $p$ is not as significant as the change with the fluctuated parameters, as shown in the previous two subsections. Due to the fact that the test planners 
mostly wish to estimate lower percentiles, the test plan is relatively robust with respect to the change in $p$. Therefore, the data from an optimally planned test with a small $p$ can be reasonably reused to estimate other lifetime percentiles if $p$ remains relatively small.

\subsection{Effect of censoring time}

Censoring in a reliability test is necessary due to the constraint of time. If there is no limitation on test time, one can test the units at use stress without extrapolation to make inferences of field reliability. By varying the right censoring time, we give the optimal ADT plans under different criteria in Table 11.

Table 11 Optimal ADT plans for various censoring time

\begin{tabular}{|c|c|c|c|c|c|c|c|c|c|c|c|c|}
\hline \multirow[b]{3}{*}{$t_{C}$} & \multicolumn{12}{|c|}{ Optimal 2-level plan } \\
\hline & \multicolumn{4}{|c|}{$[\mathrm{C} 1]$} & \multicolumn{4}{|c|}{$[\mathrm{C} 2]$} & \multicolumn{4}{|c|}{ [C3] } \\
\hline & $x_{1}$ & $x_{2}$ & $\pi_{1}$ & $\pi_{2}$ & $x_{1}$ & $x_{2}$ & $\pi_{1}$ & $\pi_{2}$ & $x_{1}$ & $x_{2}$ & $\pi_{1}$ & $\pi_{2}$ \\
\hline 750hrs (1 mo.s) & 0.56 & 1 & 0.50 & 0.50 & 0.40 & 1 & 0.82 & 0.18 & 0.43 & 1 & 0.89 & 0.11 \\
\hline $1500 \mathrm{hrs}(2 \mathrm{mo.s})$ & 0.55 & 1 & 0.51 & 0.49 & 0.39 & 1 & 0.82 & 0.18 & 0.42 & 1 & 0.89 & 0.11 \\
\hline 7500 hrs (10 mo.s) & 0.50 & 1 & 0.54 & 0.46 & 0.35 & 1 & 0.79 & 0.21 & 0.37 & 1 & 0.89 & 0.12 \\
\hline $750 \mathrm{k}$ hrs $(8 \mathrm{yr} . \mathrm{s})$ & 0 & 1 & 0.60 & 0.40 & 0 & 1 & 0.60 & 0.40 & 0 & 1 & 1 & 0 \\
\hline
\end{tabular}

As expected, larger censoring time results in a reduced optimal lower stress. Furthermore, when the censoring time is extremely large, such as 8 years in the table, the optimal lower stress level is set at use stress. For [C1] and [C2], there are still a proportion of $40 \%$ test units allocated to the lower stress under a very large $t_{C}$ because these criteria consider the variability of all parameters. In contrast, as [C3] concerns about the lifetime percentile at use stress, when the censoring time is large enough, all the test units are allocated to the lower stress in the optimal plan.

\section{Concluding remarks}

The paper has investigated the optimal constant-stress ADT plan for products subject to competing degradation and shock failures. During the test, it is assumed that traumatic shocks may occur and terminate the process of the degradation measurement. A novel ADT model based on competing failure modes is employed to derive the Fisher information for the unknown parameters. Large sample approximation provides tractable ways to optimize ADT 
plans easily by avoiding running computationally intense simulations to evaluate the plans. We use three common optimality criteria to optimize the ADT plans. A real example of LED lamps from previous literature is used to illustrate the method. The result shows that the presence of random shock failures considerably influences the optimal ADT plans under three criteria. The general equivalence theorems are used to verify the optimality of the 2-level plans. We also study different rules of three-level compromise plans, and we suggest $10 \%$ or $20 \%$ allocation rule to the middle stress in such problems. Furthermore, the general sensitivity analysis shows that the optimal plans are fairly robust under slight errors in pilot parameters. However, we have to emphasize that the parameters of shock failure models should be carefully specified.

The ADT model described in the paper can also be extended to step-stress ADT (SSADT), which is more appropriate if the test units are very limited. In addition, models incorporating multiple dependent shocks and degradation failures could be adopted in the ADT planning framework, where copula function can be applied in model construction, and revealing asymptotic properties of unknown parameters are challenging and of interest to investigate.

\section{Appendix A: Elements of the information matrix}

Following Eq. (17), the other elements in the information matrix are given by

$$
\begin{aligned}
\mathrm{E}\left(-\frac{\partial^{2} \ell(\boldsymbol{\theta})}{\partial \alpha_{1} \partial \beta_{1}}\right) & =\mathrm{E}\left(-\frac{\partial^{2} \ell(\boldsymbol{\theta})}{\partial \beta_{1} \partial \alpha_{1}}\right)=\sum_{j=1}^{J} N_{j} x_{j} \mathcal{F}_{j} \\
\mathrm{E}\left(-\frac{\partial^{2} \ell(\boldsymbol{\theta})}{\partial \beta_{1}^{2}}\right) & =\sum_{j=1}^{J} N_{j} x_{j}^{2} \mathcal{F}_{j} \\
\mathrm{E}\left(-\frac{\partial^{2} \ell(\boldsymbol{\theta})}{\partial \sigma^{2}}\right) & =\sum_{j=1}^{J} \sum_{i=1}^{N_{j}}\left\{\sum _ { l = 2 } ^ { K } \left\{\left\{\exp \left[-\lambda\left(x_{j}\right)(l-1) \Delta t\right]-\exp \left[-\lambda\left(x_{j}\right) l \Delta t\right]\right\} \times 2(l\right.\right. \\
& \left.\left.-1) \frac{1}{\sigma^{2}}\right\}+\exp \left[-\lambda\left(x_{j}\right) K \Delta t\right] 2 K \frac{1}{\sigma^{2}}\right\} \\
\mathrm{E}\left(-\frac{\partial^{2} \ell(\boldsymbol{\theta})}{\partial \alpha_{2}^{2}}\right) & =\sum_{j=1}^{J} \sum_{i=1}^{N_{j}}\left\{\frac{\Delta t \lambda\left(x_{j}\right) \exp \left[-\lambda\left(x_{j}\right) \Delta t\right]}{1-\exp \left[-\lambda\left(x_{j}\right) \Delta t\right]}\left[1-\Delta t \lambda\left(x_{j}\right)+\exp \left[-\lambda\left(x_{j}\right) \Delta t\right]\right]\right. \\
& +\sum_{l=2}^{K}\left\{\left\{\exp \left[-\lambda\left(x_{j}\right)(l-1) \Delta t\right]-\exp \left[-\lambda\left(x_{j}\right) l \Delta t\right]\right\}\right. \\
& \left.\left.\times \frac{\lambda\left(x_{j}\right) \Delta t A_{l}}{\left[1-\exp \left[-\lambda\left(x_{j}\right) \Delta t\right]\right]}\right\}+\exp \left(-\lambda\left(x_{j}\right) K \Delta t\right) \lambda\left(x_{j}\right) K \Delta t\right\}=\sum_{j=1}^{J} N_{j} \mathcal{F}_{j}^{\prime}
\end{aligned}
$$


where

$$
\begin{gathered}
A_{l}=\exp \left[-2 \lambda\left(x_{j}\right) \Delta t\right]-\exp \left[-\lambda\left(x_{j}\right) \Delta t\right]+2 l \exp \left[-\lambda\left(x_{j}\right) \Delta t\right]-l \exp \left[-2 \lambda\left(x_{j}\right) l \Delta t\right] \\
+\lambda\left(x_{j}\right) \Delta t \exp \left[-\lambda\left(x_{j}\right) l \Delta t\right]-l
\end{gathered}
$$

and

$$
\begin{aligned}
& \mathrm{E}\left(-\frac{\partial^{2} \ell(\boldsymbol{\theta})}{\partial \alpha_{2} \partial \beta_{2}}\right)=\mathrm{E}\left(-\frac{\partial^{2} \ell(\boldsymbol{\theta})}{\partial \beta_{2} \partial \alpha_{2}}\right)=\sum_{j=1}^{J} N_{j} x_{j} \mathcal{F}_{j}^{\prime} \\
& \mathrm{E}\left(-\frac{\partial^{2} \ell(\boldsymbol{\theta})}{\partial \beta_{2}^{2}}\right)=\sum_{j=1}^{J} N_{j} x_{j}^{2} \mathcal{F}_{j}^{\prime}
\end{aligned}
$$

\section{Appendix B: Elements of $\boldsymbol{H}(\boldsymbol{\theta})$}

For an arbitrary time $t$, we have

$$
\begin{aligned}
\frac{\partial F_{T}(t)}{\partial \alpha_{1}}=-\eta & (0) \exp (-\lambda(0) t)\left\{-\phi\left[\sqrt{\frac{1}{\sigma^{2} t}}(\eta(0) t-D)\right] \sqrt{\frac{t}{\sigma^{2}}}\right. \\
& -\frac{2 D}{\sigma^{2}} \exp \left(\frac{2 \eta(0) D}{\sigma^{2}}\right) \Phi\left[-\sqrt{\frac{1}{\sigma^{2} t}}(\eta(0) t+D)\right] \\
& \left.+\exp \left(\frac{2 \eta(0) D}{\sigma^{2}}\right) \phi\left[-\sqrt{\frac{1}{\sigma^{2} t}}(\eta(0) t+D)\right] \sqrt{\frac{t}{\sigma^{2}}}\right\}
\end{aligned}
$$

$\frac{\partial F_{T}(t)}{\partial \beta_{1}}=0$

$$
\begin{aligned}
\frac{\partial F_{T}(t)}{\partial \sigma}=- & \exp (-\lambda(0) t)\left\{\frac{1}{\sigma^{2}} \phi\left[\sqrt{\frac{1}{\sigma^{2} t}}(\eta(0) t-D)\right] \sqrt{\frac{1}{t}}(\eta(0) t-D)\right. \\
& +\frac{4 \eta(0) D}{\sigma^{3}} \exp \left(\frac{2 \eta(0) D}{\sigma^{2}}\right) \Phi\left[-\sqrt{\frac{1}{\sigma^{2} t}}(\eta(0) t+D)\right] \\
& \left.-\frac{1}{\sigma^{2}} \exp \left(\frac{2 \eta(0) D}{\sigma^{2}}\right) \phi\left[-\sqrt{\frac{1}{\sigma^{2} t}}(\eta(0) t+D)\right] \sqrt{\frac{1}{t}}(\eta(0) t-D)\right\}
\end{aligned}
$$

$$
\begin{array}{r}
\frac{\partial F_{T}(t)}{\partial \alpha_{2}}=\lambda(0) t \exp (-\lambda(0) t)\left\{1-\Phi\left[\sqrt{\frac{1}{\sigma^{2} t}}(\eta(0) t-D)\right]\right. \\
\left.-\exp \left(\frac{2 \eta(0) D}{\sigma^{2}}\right) \Phi\left[-\sqrt{\frac{1}{\sigma^{2} t}}(\eta(0) t+D)\right]\right\}
\end{array}
$$

$\frac{\partial F_{T}(t)}{\partial \beta_{2}}=0$ 


\section{References}

[1] R. Pan and T. Crispin, "A hierarchical modeling approach to accelerated degradation testing data analysis: A case study," Qual. Reliab. Eng. Int., vol. 27, no. 2, pp. 229-237, Mar. 2011.

[2] X. Zhao and M. Xie, "Using accelerated life tests data to predict warranty cost under imperfect repair," Comput. Ind. Eng., vol. 107, pp. 223-234, May 2017.

[3] E. A. Elsayed, "Overview of Reliability Testing," IEEE Trans. Reliab., vol. 61, no. 2, pp. 282-291, Jun. 2012.

[4] W. Q. Meeker, L. A. Escobar, and C. J. Lu, "Accelerated degradation tests: modeling and analysis," Technometrics, vol. 40, no. 2, pp. 89-99, 1998.

[5] S. Tseng and Z. Wen, "Step-stress accelerated degradation analysis for highly reliable products," J. Qual. Technol., vol. 32, no. 3, pp. 209-216, Jul. 2000.

[6] X. Xiao and Z. Ye, "Optimal design for destructive degradation tests with random initial degradation values using the Wiener process," IEEE Trans. Reliab., vol. 65, no. 3, pp. $1-16,2016$.

[7] H. Lim and B.-J. Yum, "Optimal design of accelerated degradation tests based on Wiener process models," J. Appl. Stat., vol. 38, no. 2, pp. 309-325, Feb. 2011.

[8] C.-H. Hu, M.-Y. Lee, and J. Tang, "Optimum step-stress accelerated degradation test for Wiener degradation process under constraints," Eur. J. Oper. Res., vol. 241, no. 2, pp. 412-421, Mar. 2015.

[9] C.-C. Tsai, S.-T. Tseng, and N. Balakrishnan, "Optimal design for degradation tests based on gamma processes with random effects," IEEE Trans. Reliab., vol. 61, no. 2, pp. 604-613, 2012.

[10] T.-R. Tsai, W.-Y. Sung, Y. L. Lio, S. I. Chang, and J.-C. Lu, "Optimal two-variable accelerated degradation test plan for gamma degradation processes," IEEE Trans. Reliab., vol. 65, no. 1, pp. 459-468, Mar. 2016.

[11] S. T. Tseng, N. Balakrishnan, and C. C. Tsai, "Optimal step-stress accelerated degradation test plan for gamma degradation processes," IEEE Trans. Reliab., vol. 58, 
no. 4, pp. 611-618, 2009.

[12] Z. Ye, L. Chen, L. C. Tang, and M. Xie, "Accelerated degradation test planning using the inverse Gaussian process," IEEE Trans. Reliab., vol. 63, no. 3, pp. 750-763, Sep. 2014.

[13] D. S. Bai and Y. R. Chun, "Optimum simple step-stress accelerated life-tests with competing causes of failure,” IEEE Trans. Reliab., vol. 40, no. 5, pp. 622-627, 1991.

[14] F. Pascual, "Accelerated life test planning with independent weibull competing risks," IEEE Trans. Reliab., vol. 57, no. 3, pp. 435-444, Sep. 2008.

[15] F. Pascual, "Accelerated life test planning with independent lognormal competing risks,” J. Stat. Plan. Inference, vol. 140, no. 4, pp. 1089-1100, Apr. 2010.

[16] X. Liu and L.-C. Tang, "Accelerated life test plans for repairable systems with multiple independent risks," IEEE Trans. Reliab., vol. 59, no. 1, pp. 115-127, Mar. 2010.

[17] X. Liu and W. S. Qiu, "Modeling and planning of step-stress accelerated life tests with independent competing risks," IEEE Trans. Reliab., vol. 60, no. 4, pp. 712-720, Dec. 2011.

[18] S.-J. Wu and S.-R. Huang, "Planning two or more level constant-stress accelerated life tests with competing risks," Reliab. Eng. Syst. Saf., vol. 158, no. August 2016, pp. 1-8, Feb. 2017.

[19] X. Liu, "Planning of accelerated life tests with dependent failure modes based on a gamma frailty model," Technometrics, vol. 54, no. 4, pp. 398-409, 2012.

[20] Z.-S. Ye, M. Xie, L.-C. Tang, and Y. Shen, "Degradation-based burn-in planning under competing risks," Technometrics, vol. 54, no. 2, pp. 159-168, 2012.

[21] X. Li and T. Jiang, "Optimal design for step-stress accelerated degradation testing with competing failure modes," in 2009 Annual Reliability and Maintainability Symposium, 2009, pp. 64-68.

[22] Z. Pan and Q. Sun, "Optimal design for step-stress accelerated degradation test with multiple performance characteristics based on gamma processes," Commun. Stat. Simul. Comput., vol. 43, no. 2, pp. 298-314, Jan. 2014.

[23] F. Haghighi and S. J. Bae, "Reliability estimation from linear degradation and failure time data with competing risks under a step-stress accelerated degradation test," IEEE 
Trans. Reliab., vol. 64, no. 3, pp. 960-971, 2015.

[24] K. T. Huynh, I. T. Castro, A. Barros, and C. Bérenguer, "Modeling age-based maintenance strategies with minimal repairs for systems subject to competing failure modes due to degradation and shocks," Eur. J. Oper. Res., vol. 218, no. 1, pp. 140-151, Apr. 2012.

[25] Q. Feng, K. Rafiee, E. Keedy, A. Arab, D. W. Coit, and S. Song, "Reliability and condition-based maintenance for multi-stent systems with stochastic-dependent competing risk processes," Int. J. Adv. Manuf. Technol., vol. 80, no. 9-12, pp. 20272040, Oct. 2015.

[26] B. Liu, M. Xie, Z. Xu, and W. Kuo, “An imperfect maintenance policy for missionoriented systems subject to degradation and external shocks," Comput. Ind. Eng., vol. 102, pp. 21-32, Dec. 2016.

[27] C.-H. Hu, R. D. Plante, and J. Tang, "Equivalent step-stress accelerated life tests with log-location-scale lifetime distributions under Type-I censoring," IIE Trans., vol. 47, no. 3, pp. 245-257, Jan. 2015.

[28] Z.-S. Ye and M. Xie, "Stochastic modelling and analysis of degradation for highly reliable products," Appl. Stoch. Model. Bus. Ind., vol. 31, no. 1, pp. 16-32, Jan. 2015.

[29] F. Jakob, M. Kimmelmann, and B. Bertsche, "Selection of acceleration models for test planning and model usage," IEEE Trans. Reliab., pp. 1-11, 2016.

[30] D. Han and H. K. T. Ng, "Comparison between constant-stress and step-stress accelerated life tests under Time Constraint," Nav. Res. Logist., vol. 60, no. 7, pp. 541556, Oct. 2013.

[31] P. Whittle, "Some general points in the theory of optimal experimental design," J. R. Stat. Soc. Ser. B, pp. 123-130, 1973.

[32] V. V. Fedorov, "Design of experiments for linear optimality criteria," Theory Probab. Its Appl., vol. 16, no. 1, pp. 189-195, Jan. 1971.

[33] S.-T. Tseng and I.-C. Lee, "Optimum allocation rule for accelerated degradation tests with a class of exponential-dispersion degradation models," Technometrics, vol. 58, no. 2, pp. 244-254, Apr. 2016.

[34] W. Q. Meeker, “A comparison of accelerated life test plans for weibull and lognormal 
distributions and type i censoring," Technometrics, vol. 26, no. 2, pp. 157-171, 1984.

[35] W. B. Nelson, Accelerated testing: statistical models, test plans, and data analysis, vol. 344. John Wiley \& Sons, 1990.

Xiujie Zhao received the B.E. degree in Industrial Engineering from Tsinghua University, Beijing, China, in 2013, the M.S. degree in Industrial Engineering from the Pennsylvania State University, University Park, PA, USA, in 2015.

He is currently pursuing Ph.D. degree in the Department of Systems Engineering and Engineering Management, City University of Hong Kong. His research interests include accelerated reliability testing, degradation modeling, maintenance modeling and design of experiments.

Jianyu Xu received the B.S. degree in Statistics from Peking University, Beijing, China, in 2012

$\mathrm{He}$ is currently in a joint-Ph.D. program between Department of Systems Engineering and Engineering Management, City University of Hong Kong and University of Chinese Academy of Sciences, Beijing, China. His research interests include system reliability assessment and demonstration test, degradation modeling, reliability statistics.

Bin Liu received the B.S. degree in Automation from Zhejiang University, Zhejiang, China, in 2013.

He is currently completing a Ph.D. in the Department of Systems Engineering and Engineering Management, City University of Hong Kong. His research interests include reliability and maintenance modeling, importance measures with application to complex systems, and data analysis. 